\title{
TriNet Strong-Motion Data from the M 7.1 Hector Mine, California, Earthquake of 16 October 1999
}

\author{
by Vladimir Graizer, Anthony Shakal, Craig Scrivner, Egill Hauksson, \\ Jasha Polet, and Lucy Jones
}

\begin{abstract}
The $M_{\mathrm{w}} 7.1$ Hector Mine earthquake of October 16, 1999 was recorded by more than 300 stations of TriNet, which is administered cooperatively by the California Division of Mines and Geology's California Strong Motion Instrumentation Program (CDMG/CSMIP), California Institute of Technology, and the U.S. Geological Survey (USGS). The earthquake occurred in a remote part of the Mojave Desert, approximately $190 \mathrm{~km}$ northeast of downtown Los Angeles, and there were no strong-motion stations close to the surface rupture. The nearest station, Hector, is about $27 \mathrm{~km}$ north of the epicenter; it recorded a peak horizontal ground acceleration of $0.33 \mathrm{~g}$. The two next closest stations, Amboy and Joshua Tree, are to the east and south, both at epicentral distances of about $50 \mathrm{~km}$; each recorded peak ground accelerations of about $0.2 \mathrm{~g}$. The new digital instruments installed for the TriNet project recorded a large set of reliable data at epicentral distances up to 275 $\mathrm{km}$. These data can significantly improve empirical peak ground motion attenuation relationships, which are usually developed for distances only up to $100 \mathrm{~km}$ (Boore et al., 1993, 1997) because adequate data have not been available at greater distances.

Hector Mine peak ground motions demonstrate reasonable agreement with empirical attenuation relationships for acceleration. In contrast, higher than expected ground velocities and displacements were recorded at epicentral distances of about 150 to $220 \mathrm{~km}$, especially in the Los Angeles sedimentary basin, where anomalously high-amplitude displacements with periods of 6 to $7 \mathrm{sec}$ were recorded in Los Angeles, Long Beach, and other areas. These long-period surface- or basin-generated waves can have significant effects on large structures.

The $M_{\mathrm{w}} 7.3$ Landers earthquake of 1992 similarly produced strong, long-period waves in the basin. The peak ground motions produced by the Landers earthquake were on average 1.6 times higher than for the Hector Mine earthquake in the Los Angeles area.

Ground-motion data recorded by digital instruments were uniformly processed in the frequency band 0.067 to $46 \mathrm{~Hz}(0.022-15 \mathrm{sec})$. The processed data set includes records from 213 ground-response stations. In an effort to make strong-motion data available quickly to the engineering and scientific communities, important records from this event were made available by file transfer protocol (ftp) beginning the day of the earthquake.
\end{abstract}

\section{Introduction}

The $M_{\mathrm{w}} 7.1$ Hector Mine, California, earthquake occurred on 16 October 1999 at 2:46 a.m. PDT in a remote part of the Mojave Desert area, approximately $190 \mathrm{~km}$ northeast of downtown Los Angeles. The hypocenter is located at $34.59^{\circ} \mathrm{N}$ and $116.27^{\circ} \mathrm{W}$ at a depth of $5 \mathrm{~km}$ (Hauksson et al., 2002). The earthquake produced approximately $45 \mathrm{~km}$ of surface faulting along part of the Bullion fault and the previously unnamed Lavic Lake fault, as well as parts of several other faults (Treiman et al., 2002). The fault mechanism was right-lateral strike slip, with an observed average slip of 250 to $300 \mathrm{~cm}$ across the entire fault zone and vertical displacements of $100 \mathrm{~cm}$ or less (Trelman et al., 2002). The rupture was located approximately 20 to $30 \mathrm{~km}$ east-northeast of the $1992 M_{\mathrm{w}} 7.3$ Landers fault rupture.

The Hector Mine earthquake was recorded by more than 300 strong-motion stations of TriNet, which is administered 
by the California Strong Motion Instrumentation Program (Division of Mines and Geology), California Institute of Technology, and the U.S. Geological Survey (Mori et al., 1999). The station HEC near Hector was the closest seismic station, located approximately $27 \mathrm{~km}$ north of the epicenter (10.7 km from the nearest part of the rupture); it recorded a peak horizontal ground acceleration of $0.33 \mathrm{~g}$. The two other stations closest to the epicenter were Amboy and Joshua Tree. They are located to the east and south, at epicentral distances of about $50 \mathrm{~km}$, and recorded peak ground accelerations of 0.18 and $0.19 g$, respectively. Maximum values of ground acceleration, velocity, and displacement recorded by the TriNet ground-response stations are listed in Table 1. To make comparison of processed data easier, all digital records were processed uniformly in the same frequency band: 0.067 to $46 \mathrm{~Hz}(0.022-15 \mathrm{sec})$. The data set includes records from 213 ground-response stations.

In contrast to the strong-motion data sets recorded largely by analog instruments during the 1992 Landers and the 1994 Northridge earthquakes (e.g., Shakal et al., 1992, 1994; Cramer and Darragh, 1994), most of the data recorded during the Hector Mine earthquake were obtained using digital instruments. The second main difference is that no largeamplitude, near-source ground motions were recorded during the Hector Mine event, because of the sparse distribution of stations in the Mojave Desert area. The new digital instrumentation installed in the TriNet project recorded a large set of reliable data at epicentral distances up to $275 \mathrm{~km}$. The recorded strong-motion data can be used in earthquake source- and wave-propagation modeling and in engineering analysis of ground shaking. These data can also be used to significantly improve empirical peak horizontal ground motion attenuation relationships, which are usually developed for distances of only $100 \mathrm{~km}$ or less (Boore et al., 1993, 1997) because not enough reliable data have been available for greater distances.

\section{Highlights of Ground-Response Strong-Motion Records}

A list of all processed records from the TriNet groundresponse stations used in this article is given in Table 1. The stations are listed in order of increasing epicentral distance. The processed time series for acceleration, velocity, and displacement at six ground-response stations were selected to highlight important features of the data set. The records from Big Bear Lake-Fire Station (epicentral distance $r=68 \mathrm{~km}$ ), Wrightwood-Nielson Ranch $(r=121 \mathrm{~km})$, Altadena-Eaton Canyon Park $(r=174 \mathrm{~km})$, Downey-County Maintenance Bldg. $(r=190 \mathrm{~km})$, Long Beach-Los Coyotes and Stearns $(r=193 \mathrm{~km})$, and Los Angeles-116th Street School ( $r=$ $197 \mathrm{~km}$ ) are shown in Figure 1. For all records, 80-sec time intervals are shown.

Peak ground acceleration generally decreases with increasing epicentral distance at the selected stations (Table 2 ), but the table also shows that peak ground velocities
(PGV) and peak ground displacements (PGD) do not follow a simple attenuation pattern.

Displacement waveforms at the first three stations are similar in shape, and are characterized by approximately 15 to $20 \mathrm{sec}$ of relatively simple motion (Fig. 1). This motion may be associated with the $S$ wave coming from the earthquake source, with a period of approximately 6 to $8 \mathrm{sec}$. The maximum amplitude of this displacement generally decreases with increasing epicentral distance to about $100 \mathrm{~km}$, beyond which it remains nearly constant at about $4 \mathrm{~cm}$. This simple type of ground displacement also can be seen at many stations at epicentral distances up to $240 \mathrm{~km}$ (e.g., Pacoima, Sylmar, Newhall, Tarzana, and Moorpark; see Table 1). According to Dreger and Kaverina (2000a,b), the major part of the seismic moment was released within $20 \mathrm{sec}$ during the Hector Mine earthquake. This time interval corresponds to the duration of the recorded $S$-wave motion.

The long-period part of the velocity and displacement waveforms are significantly amplified in the Long Beach and Los Angeles areas, with displacements up to $15 \mathrm{~cm}$ and corresponding maximum horizontal velocities up to $13 \mathrm{~cm} / \mathrm{sec}$. Those are approximately the same levels as at the closest stations. Ground motions with long-duration wavetrains after the $S$-wave arrival were recorded at a large number of stations. Long duration (40-60 sec) and high amplitudes of long-period $(6-8 \mathrm{sec})$ waves may be due to the basin waves or surface waves generated in the basin. These waves have approximately the same periods as the source-generated $S$ waves.

Response spectra (5\% damped; Fig. 2a) of the N-S components of acceleration calculated for the three closest stations (Big Bear, Wrightwood, and Altadena; Fig. 1a-c) have maximum amplitudes at periods around 0.2 to $0.7 \mathrm{sec}$ (frequencies 1.4-5.0 Hz), with much lower amplitudes at longer periods. In contrast to those three stations, spectral accelerations at the stations in the Los Angeles basin (Downey, Long Beach, and Los Angeles; Fig. 2b) contain a very strong long-period $(5-8 \mathrm{sec})$ component. This motion can also be seen in the time domain (Fig. 1d-f).

Some stations in other areas also demonstrate relatively long duration of long-period motion. For example, the two stations on deep sediments in the San Bernardino area (San Bernardino-E and Hospitality, $r=111 \mathrm{~km}$, and San Bernardino-Mountain. View and Cluster, $r=108 \mathrm{~km}$ ) demonstrate up to $40 \mathrm{sec}$ of long-period (5-6 sec), highamplitude motions. Ground motions at other stations over shallow sediments in the San Bernardino area (San Bernardino-Highland and Del Rosa, $r=104 \mathrm{~km}$; Highland, $r=102 \mathrm{~km}$; and Rialto, $r=117 \mathrm{~km}$ ) are more similar in shape to the motion at the Big Bear, Wrightwood, and Altadena stations, with the duration of main motion up to $20 \mathrm{sec}$ and much lower amplitudes (see Table 1). These large variations in strong motion may be due to the differences in wave propagation path and site geology, especially the varying thickness and depth of the relatively soft sedimentary basin layers. Records in Salton City (148 km) and El Centro 
Table 1

Peak Horizontal Ground Motions at TriNet Stations during the Hector Mine Earthquake

\begin{tabular}{|c|c|c|c|c|c|c|c|c|c|}
\hline $\begin{array}{l}\text { Station } \\
\text { ID }\end{array}$ & Station Name & Network & Lat & Long & $\begin{array}{l}\text { Epi Dist } \\
(\mathrm{km})\end{array}$ & $\begin{array}{l}\text { Fault Dist } \\
\quad(\mathrm{km})\end{array}$ & $\begin{array}{l}\text { Accel } \\
(\mathrm{g})\end{array}$ & $\begin{array}{c}\mathrm{Vel} \\
(\mathrm{cm} / \mathrm{sec})\end{array}$ & $\begin{array}{l}\text { Displ } \\
(\mathrm{cm})\end{array}$ \\
\hline HEC & Hector & SCSN & 34.83 & 116.33 & 27.2 & 10.7 & 0.330 & 44.5 & 13.4 \\
\hline 21081 & Amboy & CDMG & 34.56 & 115.74 & 48.5 & 45.8 & 0.180 & 27.3 & 14.0 \\
\hline 22170 & Joshua Tree-Fire Station & CDMG & 34.13 & 116.31 & 51.2 & 22.6 & 0.190 & 23.2 & 6.5 \\
\hline 22791 & Big Bear Lake-Fire Station & CDMG & 34.24 & 116.87 & 67.5 & 65.3 & 0.170 & 13.3 & 9.2 \\
\hline 22161 & Twentynine Palms-Joshua Tree N.M. & CDMG & 34.02 & 116.01 & 67.5 & 40.1 & 0.060 & 6.6 & 5.3 \\
\hline $22 \mathrm{~T} 04$ & Heart Bar State Park & CDMG & 34.16 & 116.80 & 68.1 & 60.9 & 0.082 & 11.6 & 8.3 \\
\hline 12149 & Desert Hot Springs-Fire Station & CDMG & 33.96 & 116.51 & 73.1 & 47.1 & 0.082 & 7.5 & 3.8 \\
\hline 12647 & Joshua Tree N.M.-Keys View & CDMG & 33.92 & 116.17 & 74.3 & 47.7 & 0.089 & 7.9 & 4.8 \\
\hline 32075 & Baker-Fire Station & CDMG & 35.27 & 116.07 & 77.8 & 54.7 & 0.130 & 10.1 & 3.1 \\
\hline 23559 & Barstow-Vineyard \& H St. & CDMG & 34.89 & 117.05 & 78.4 & 57.9 & 0.070 & 7.8 & 3.9 \\
\hline DAN & Danby & SCSN & 34.64 & 115.38 & 81.7 & 77.0 & 0.130 & 10.3 & 6.6 \\
\hline 32577 & Fort Irwin & CDMG & 35.27 & 116.68 & 84.2 & 59.9 & 0.130 & 14.3 & 7.9 \\
\hline 12630 & Snow Creek & CDMG & 33.89 & 116.68 & 86.7 & 65.2 & 0.029 & 3.8 & 3.1 \\
\hline 12674 & Banning-Twin Pines Rd. & CDMG & 33.87 & 116.82 & 94.9 & 77.7 & 0.021 & 4.0 & 4.0 \\
\hline 12543 & Indio-Riverside Co. Fairgrounds & CDMG & 33.72 & 116.22 & 97.2 & 71.1 & 0.120 & 19.0 & 13.6 \\
\hline 12026 & Indio-Coachella Canal & CDMG & 33.72 & 116.15 & 97.4 & 70.8 & 0.124 & 13.7 & 7.7 \\
\hline 12919 & Beaumont-6th \& Maple & CDMG & 33.93 & 116.97 & 97.7 & 87.3 & 0.062 & 13.4 & 10.7 \\
\hline 23583 & Hesperia-4th \& Palm & CDMG & 34.40 & 117.31 & 98.0 & 90.6 & 0.063 & 10.1 & 5.5 \\
\hline HLN & Highland & SCSN & 34.12 & 117.22 & 101.6 & 96.0 & 0.036 & 8.8 & 6.4 \\
\hline 23897 & San Bernardino-Highland \& Del Rosa & CDMG & 34.13 & 117.25 & 103.6 & 102.6 & 0.045 & 8.3 & 7.5 \\
\hline 12618 & San Jacinto-Soboba A & CDMG & 33.80 & 116.88 & 103.9 & 85.5 & 0.061 & 6.9 & 3.9 \\
\hline 12624 & Lake Cahuilia-County Park & CDMG & 33.63 & 116.28 & 106.7 & 81.2 & 0.025 & 4.0 & 2.0 \\
\hline 23732 & San Bernardino-Devil Canyon Penstock & CDMG & 34.19 & 117.33 & 107.1 & 104.8 & 0.023 & 7.4 & 6.1 \\
\hline 23780 & San Bernardino-Mtn. View \& Cluster & CDMG & 34.10 & 117.29 & 108.3 & 105.3 & 0.061 & 18.3 & 9.3 \\
\hline 23898 & San Bernardino-Medical Cntr \& Highland & CDMG & 34.13 & 117.32 & 109.1 & 108.1 & 0.060 & 10.1 & 9.4 \\
\hline 12673 & San Jacinto-CDF Fire Station & CDMG & 33.79 & 116.96 & 109.3 & 92.9 & 0.060 & 18.2 & 16.0 \\
\hline 23542 & San Bernardino-E \& Hospitality & CDMG & 34.06 & 117.29 & 110.6 & 107.8 & 0.072 & 16.0 & 10.9 \\
\hline 13927 & Moreno Valley-Alessandro \& Moreno Bch & CDMG & 33.92 & 117.17 & 111.5 & 103.8 & 0.062 & 5.7 & 5.9 \\
\hline 12923 & Hernet-Acacia \& Stanford & CDMG & 33.74 & 116.93 & 111.9 & 92.9 & 0.063 & 13.3 & 5.8 \\
\hline 13926 & Moreno Valley-Hwy 60 \& Heacock & CDMG & 33.94 & 117.25 & 115.4 & 109.1 & 0.050 & 6.0 & 5.4 \\
\hline 23597 & Phelan-Wilson Ranch Road & CDMG & 34.47 & 117.52 & 115.6 & 105.5 & 0.073 & 7.5 & 3.7 \\
\hline 12331 & Hemet-Stetson Ave Fire Station & CDMG & 33.73 & 116.98 & 115.7 & 97.6 & 0.067 & 6.8 & 3.7 \\
\hline 11825 & Mecca-CVWD Yard & CDMG & 33.56 & 115.99 & 116.8 & 89.5 & 0.100 & 19.9 & 14.8 \\
\hline 23899 & Rialto-I10 \& Cedar & CDMG & 34.07 & 117.40 & 118.8 & 117.0 & 0.036 & 5.2 & 4.9 \\
\hline 13915 & Riverside-I215 \& 3rd & CDMG & 33.98 & 117.34 & 119.9 & 115.5 & 0.054 & 6.4 & 4.5 \\
\hline 23912 & Fontana-Arrow \& Sierra & CDMG & 34.10 & 117.43 & 120.1 & 118.5 & 0.046 & 6.0 & 5.3 \\
\hline FON & Fontana & SCSN & 34.10 & 117.44 & 120.5 & 118.8 & 0.040 & 6.4 & 5.3 \\
\hline 23573 & Wrightwood-Nielson Ranch & CDMG & 34.31 & 117.54 & 121.1 & 114.2 & 0.054 & 5.0 & 4.4 \\
\hline 13924 & Homeland-Hwy 74 \& Sultanas & CDMG & 33.75 & 117.13 & 122.3 & 110.7 & 0.040 & 3.2 & 3.9 \\
\hline 13916 & Riverside-Van Buren \& Trautwein & CDMG & 33.90 & 117.32 & 123.7 & 117.2 & 0.034 & 4.1 & 4.2 \\
\hline 13928 & Perris-San Jacinto \& C St. & CDMG & 33.79 & 117.23 & 125.6 & 115.9 & 0.018 & 4.0 & 3.8 \\
\hline 13930 & Sun City-I215 \& McCall Blvd & CDMG & 33.72 & 117.19 & 128.8 & 117.4 & 0.041 & 3.9 & 3.5 \\
\hline 13123 & Riverside-Airport & CDMG & 33.95 & 117.45 & 129.4 & 125.4 & 0.025 & 4.1 & 4.0 \\
\hline 12626 & Desert Shores & CDMG & 33.43 & 116.08 & 130.3 & 103.4 & 0.039 & 3.0 & 2.0 \\
\hline 13921 & Riverside-Limonite \& Downey & CDMG & 33.97 & 117.49 & 131.1 & 127.9 & 0.029 & 4.6 & 4.4 \\
\hline 23585 & Palmdale-Black Butte & CDMG & 34.59 & 117.73 & 133.8 & 120.7 & 0.019 & 2.8 & 1.8 \\
\hline 13913 & Riverside-Hole \& La Sierra & CDMG & 33.92 & 117.49 & 134.6 & 130.3 & 0.036 & 4.3 & 3.9 \\
\hline 13929 & Menifee Valley-Murrieta \& Scott & CDMG & 33.65 & 117.21 & 135.4 & 123.2 & 0.032 & 3.1 & 3.6 \\
\hline 11591 & North Shore-Durmid & CDMG & 33.42 & 115.83 & 135.9 & 108.4 & 0.075 & 15.1 & 10.4 \\
\hline 23896 & Ontario-I10 \& 4th & CDMG & 34.08 & 117.62 & 136.7 & 133.8 & 0.031 & 7.2 & 5.4 \\
\hline 23590 & Wrightwood-Jackson Flat & CDMG & 34.38 & 117.74 & 136.7 & 127.5 & 0.045 & 6.4 & 3.7 \\
\hline 11627 & Bombay Beach-Bertram & CDMG & 33.40 & 115.78 & 140.0 & 112.5 & 0.035 & 11.2 & 10.4 \\
\hline 23895 & Ontario-4th \& Mountain & CDMG & 34.08 & 117.67 & 140.9 & 137.6 & 0.034 & 8.2 & 7.5 \\
\hline 13918 & Norco-1st \& Hamner & CDMG & 33.90 & 117.56 & 141.2 & 137.2 & 0.016 & 4.3 & 4.0 \\
\hline 13922 & Lake Elsinore-Graham \& Poe & CDMG & 33.67 & 117.33 & 141.5 & 131.1 & 0.034 & 5.4 & 5.4 \\
\hline 13726 & Corona-Calif. Ave. & CDMG & 33.85 & 117.54 & 142.6 & 137.5 & 0.031 & 4.8 & 4.1 \\
\hline CRN & Corona & SCSN & 33.88 & 117.56 & 143.0 & 138.4 & 0.030 & 5.0 & 4.2 \\
\hline 11684 & Frink & CDMG & 33.40 & 115.66 & 143.8 & 116.5 & 0.072 & 11.7 & 5.4 \\
\hline 13172 & Temecula-CDF & CDMG & 33.50 & 117.15 & 146.0 & 123.5 & 0.057 & 9.0 & 3.9 \\
\hline 11628 & Salton City & CDMG & 33.28 & 115.98 & 147.7 & 120.6 & 0.055 & 7.1 & 4.4 \\
\hline
\end{tabular}


Table 1

Continued

\begin{tabular}{|c|c|c|c|c|c|c|c|c|c|}
\hline $\begin{array}{l}\text { Station } \\
\text { ID }\end{array}$ & Station Name & Network & Lat & Long & $\begin{array}{l}\text { Epi Dist } \\
(\mathrm{km})\end{array}$ & $\begin{array}{l}\text { Fault Dist } \\
(\mathrm{km})\end{array}$ & $\begin{array}{c}\text { Accel } \\
(\mathrm{g})\end{array}$ & $\begin{array}{c}\mathrm{Vel} \\
(\mathrm{cm} / \mathrm{sec})\end{array}$ & $\begin{array}{l}\text { Displ } \\
(\mathrm{cm})\end{array}$ \\
\hline 23525 & Pomona-4th \& Locust & CDMG & 34.06 & 117.75 & 148.4 & 144.8 & 0.037 & 8.4 & 6.3 \\
\hline 23837 & Pomona-Orange Grove \& Fairplex & CDMG & 34.06 & 117.78 & 151.1 & 147.1 & 0.034 & 6.9 & 5.9 \\
\hline 23836 & Glendora-Gladstone \& Sunflower & CDMG & 34.11 & 117.85 & 154.7 & 149.2 & 0.038 & 6.6 & 6.0 \\
\hline 13122 & Featherly Park-Maint. Bldg. & CDMG & 33.87 & 117.71 & 154.8 & 151.4 & 0.041 & 6.9 & 6.7 \\
\hline 23595 & Littlerock-Brainard Canyon & CDMG & 34.49 & 117.98 & 157.4 & 145.6 & 0.034 & 3.6 & 2.7 \\
\hline LTR & Littlerock & SCSN & 34.52 & 117.99 & 158.1 & 145.7 & 0.054 & 4.3 & 2.4 \\
\hline 13882 & Olinda-Carbon Canyon Rd & CDMG & 33.93 & 117.80 & 159.2 & 157.1 & 0.042 & 8.4 & 7.4 \\
\hline 23774 & West Covina-Kaiser Grounds & CDMG & 34.07 & 117.94 & 164.0 & 158.8 & 0.027 & 11.1 & 12.1 \\
\hline 13849 & Anaheim-Lakeview \& Riverdale & CDMG & 33.85 & 117.82 & 164.3 & 161.3 & 0.056 & 13.1 & 10.5 \\
\hline 23843 & La Puente-Amar \& Hacienda & CDMG & 34.04 & 117.95 & 166.4 & 161.7 & 0.040 & 13.7 & 10.6 \\
\hline 13873 & Brea-Central Av Caltrans Yard & CDMG & 33.93 & 117.90 & 166.6 & 163.7 & 0.026 & 10.5 & 8.3 \\
\hline 23842 & City of Industry-Gale \& Azusa & CDMG & 34.00 & 117.93 & 166.6 & 162.6 & 0.048 & 9.8 & 7.3 \\
\hline 13883 & Anaheim-Kraemer \& La Palma & CDMG & 33.85 & 117.86 & 167.5 & 164.8 & 0.040 & 12.4 & 11.0 \\
\hline 23773 & Baldwin Park-Kaiser Grounds & CDMG & 34.07 & 117.98 & 168.0 & 162.7 & 0.025 & 14.4 & 12.8 \\
\hline 13878 & Fullerton-CSU Fullerton Grounds & CDMG & 33.89 & 117.89 & 168.0 & 165.8 & 0.042 & 12.2 & 11.2 \\
\hline 13888 & Orange-Shaffer \& Taft & CDMG & 33.82 & 117.85 & 168.8 & 165.5 & 0.029 & 9.9 & 10.3 \\
\hline 13875 & Orange-Hwy 22/Hwy 55 Grounds & CDMG & 33.78 & 117.83 & 169.9 & 165.8 & 0.036 & 10.4 & 6.4 \\
\hline OGC & Orange & SCSN & 33.79 & 117.84 & 170.2 & 166.3 & 0.045 & 9.8 & 7.4 \\
\hline 13880 & Fullerton-Hermosa \& Harbor & CDMG & 33.91 & 117.93 & 170.5 & 167.8 & 0.030 & 11.8 & 11.0 \\
\hline 13881 & La Habra-La Habra \& Monte Vista & CDMG & 33.93 & 117.96 & 171.5 & 168.3 & 0.036 & 11.7 & 8.7 \\
\hline FUL & Fullerton & SCSN & 33.87 & 117.92 & 171.8 & 169.7 & 0.040 & 17.1 & 17.6 \\
\hline 24850 & Whittler-Workman Mill Rd \& Pomona Fwy & CDMG & 34.03 & 118.02 & 172.5 & 167.5 & 0.038 & 11.0 & 8.9 \\
\hline 13889 & Santa Ana-I5 \& 4th St & CDMG & 33.75 & 117.84 & 172.6 & 168.1 & 0.029 & 8.7 & 7.6 \\
\hline 24402 & Altadena-Eaton Canyon Park & CDMG & 34.18 & 118.10 & 174.1 & 166.6 & 0.037 & 4.9 & 4.0 \\
\hline 13893 & Orange-I5 \& Chapman & CDMG & 33.78 & 117.89 & 174.1 & 170.4 & 0.039 & 13.6 & 9.2 \\
\hline 14840 & Whittler-Scott \& Whittler & CDMG & 33.95 & 118.00 & 174.6 & 170.9 & 0.042 & 11.5 & 10.2 \\
\hline 13879 & Fullerton-Valencia \& Brookhurst & CDMG & 33.87 & 117.96 & 175.0 & 172.7 & 0.046 & 16.7 & 12.0 \\
\hline KIK & Pasadena-Kinemetrics & SCSN & 34.15 & 118.10 & 175.4 & 168.2 & 0.023 & 5.7 & 4.0 \\
\hline 13894 & Garden Grove-Chapman \& West & CDMG & 33.79 & 117.92 & 176.3 & 173.0 & 0.043 & 12.8 & 11.3 \\
\hline 13890 & Santa Ana-1st \& Franklin & CDMG & 33.74 & 117.89 & 176.8 & 172.6 & 0.038 & 13.1 & 10.0 \\
\hline 24576 & Anaverde Valley-City Ranch & CDMG & 34.58 & 118.20 & 177.0 & 163.5 & 0.038 & 3.7 & 1.6 \\
\hline 13884 & Garden Grove-Hwy 22 \& Harbor Blvd & CDMG & 33.77 & 117.92 & 177.3 & 173.6 & 0.035 & 12.4 & 11.1 \\
\hline 13892 & Santa Ana-Macarthur \& Bristol & CDMG & 33.70 & 117.88 & 178.3 & 173.3 & 0.039 & 7.9 & 6.9 \\
\hline SJU & San Juan Capistrano & SCSN & 33.49 & 117.68 & 178.7 & 169.2 & 0.026 & 8.1 & 6.8 \\
\hline 24841 & Pico Rivera-Durfee \& Whittler & CDMG & 34.00 & 118.08 & 178.7 & 173.8 & 0.021 & 8.5 & 7.2 \\
\hline 24401 & San Marino-SW Academy & CDMG & 34.12 & 118.13 & 179.1 & 172.3 & 0.018 & 3.6 & 3.6 \\
\hline 13891 & Santa Ana-Warner \& Greenville & CDMG & 33.72 & 117.90 & 179.2 & 174.6 & 0.050 & 10.6 & 7.9 \\
\hline 13795 & Capistrano Beach-I5/Via Calif. Bridge & CDMG & 33.47 & 117.67 & 179.4 & 169.4 & 0.032 & 11.1 & 7.6 \\
\hline 24691 & Pasadena-Fair Oaks \& Walnut & CDMG & 34.15 & 118.15 & 179.7 & 172.4 & 0.040 & 4.9 & 3.2 \\
\hline 13886 & Garden Grove-Chapman \& Gilbert & CDMG & 33.79 & 117.97 & 179.9 & 176.9 & 0.034 & 15.0 & 12.6 \\
\hline 24692 & Pasadena-Orange Grove \& Pasadena & CDMG & 34.15 & 118.16 & 180.2 & 172.9 & 0.040 & 5.3 & 3.3 \\
\hline 13885 & Garden Grove-Brookhurst \& Westminster & CDMG & 33.76 & 117.96 & 180.8 & 177.2 & 0.032 & 11.9 & 12.8 \\
\hline 24461 & Alhambra-Fremont School & CDMG & 34.07 & 118.15 & 182.4 & 176.2 & 0.024 & 5.7 & 5.5 \\
\hline 13333 & Oceanside B-Fire Station & CDMG & 33.20 & 117.33 & 182.7 & 153.6 & 0.014 & 3.3 & 2.5 \\
\hline 14828 & Downey-Florence \& I605 & CDMG & 33.94 & 118.10 & 183.2 & 179.0 & 0.041 & 10.0 & 8.8 \\
\hline 24468 & Los Angeles-CSULA Admin. Building & CDMG & 34.07 & 118.17 & 184.1 & 177.8 & 0.040 & 10.0 & 8.9 \\
\hline 13160 & Newport Beach-Irvine Ave Fire Sta & CDMG & 33.63 & 117.90 & 184.2 & 178.4 & 0.030 & 9.1 & 8.0 \\
\hline 24592 & Los Angeles-City Terrace & CDMG & 34.05 & 118.17 & 184.8 & 178.7 & 0.038 & 6.7 & 5.3 \\
\hline 13877 & Newport Beach-Balboa Island & CDMG & 33.60 & 117.89 & 185.1 & 178.8 & 0.034 & 6.5 & 5.5 \\
\hline 24814 & Los Angeles-Colorado \& Eagle Rock & CDMG & 34.14 & 118.21 & 185.3 & 177.9 & 0.025 & 3.0 & 3.1 \\
\hline LEV & Leona Valley & SCSN & 34.61 & 118.29 & 185.4 & 171.4 & 0.050 & 3.8 & 2.0 \\
\hline 24400 & Los Angeles-Obregon Park & CDMG & 34.04 & 118.18 & 186.0 & 180.1 & 0.033 & 6.6 & 6.3 \\
\hline 14844 & Bell Gardens-Garfield \& Florence & CDMG & 33.97 & 118.15 & 186.2 & 181.4 & 0.027 & 12.3 & 10.4 \\
\hline 13887 & Huntington Beach-Adams \& Bushard & CDMG & 33.68 & 117.96 & 186.2 & 181.4 & 0.052 & 9.8 & 9.4 \\
\hline 14827 & Downey-Imperial \& Bellflower & CDMG & 33.92 & 118.13 & 186.4 & 182.3 & 0.026 & 10.5 & 10.6 \\
\hline 13876 & Newport Beach-Balboa \& Medina & CDMG & 33.60 & 117.90 & 186.4 & 180.2 & 0.026 & 6.5 & 5.1 \\
\hline 14874 & Westminster-I405/Hwy 22 Grounds & CDMG & 33.77 & 118.04 & 186.8 & 183.9 & 0.034 & 13.5 & 12.0 \\
\hline 24605 & Los Angeles-7-story Univ. Hospital Grnds & CDMG & 34.06 & 118.20 & 186.9 & 180.5 & 0.020 & 5.4 & 4.8 \\
\hline 14829 & Bellflower-Flora Vista \& Woodruff & CDMG & 33.88 & 118.12 & 187.4 & 183.8 & 0.032 & 11.1 & 12.4 \\
\hline 14830 & Lakewood-Del Amo \& Palo Verde & CDMG & 33.85 & 118.11 & 118.2 & 185.2 & 0.044 & 13.5 & 14.7 \\
\hline
\end{tabular}


Table 1

Continued

\begin{tabular}{|c|c|c|c|c|c|c|c|c|c|}
\hline $\begin{array}{l}\text { Station } \\
\text { ID }\end{array}$ & Station Name & Network & Lat & Long & $\begin{array}{c}\text { Epi Dist } \\
(\mathrm{km})\end{array}$ & $\begin{array}{l}\text { Fault Dist } \\
(\mathrm{km})\end{array}$ & $\begin{array}{l}\text { Accel } \\
\text { (g) }\end{array}$ & $\begin{array}{c}\mathrm{Vel} \\
(\mathrm{cm} / \mathrm{sec})\end{array}$ & $\begin{array}{l}\text { Displ } \\
(\mathrm{cm})\end{array}$ \\
\hline 24813 & Cypress Park-San Fernando \& Division & CDMG & 34.10 & 118.23 & 188.5 & 181.6 & 0.025 & 3.5 & 3.0 \\
\hline 14368 & Downey-County Malnt. Bldg. & CDMG & 33.92 & 118.17 & 189.7 & 185.3 & 0.033 & 13.3 & 10.9 \\
\hline 13197 & Huntington Beach-Lake St Fire Station & CDMG & 33.66 & 118.00 & 189.7 & 185.0 & 0.031 & 9.0 & 7.9 \\
\hline 14869 & Long Beach-Los Coyotes \& Palo Verde & CDMG & 33.82 & 118.11 & 189.7 & 187.1 & 0.027 & 11.5 & 11.9 \\
\hline 14825 & Huntington Park-Saturn \& Hood & CDMG & 33.98 & 118.21 & 191.0 & 185.8 & 0.030 & 12.7 & 9.9 \\
\hline 24812 & Los Angeles-Rowena \& Glendale & CDMG & 34.11 & 118.26 & 191.0 & 183.8 & 0.020 & 3.2 & 2.3 \\
\hline 24611 & Los Angeles-Temple \& Hope & CDMG & 34.06 & 118.25 & 191.2 & 184.6 & 0.026 & 4.8 & 4.5 \\
\hline 24839 & Los Angeles-1st \& Figueroa & CDMG & 34.06 & 118.25 & 191.7 & 185.2 & 0.024 & 5.3 & 4.2 \\
\hline 14872 & Long Beach-Artesia \& Orange & CDMG & 33.87 & 118.18 & 192.7 & 188.9 & 0.031 & 12.8 & 10.9 \\
\hline 14870 & Long Beach-Los Coyotes \& Steams & CDMG & 33.80 & 118.14 & 193.1 & 190.5 & 0.031 & 12.4 & 11.7 \\
\hline 24838 & Los Angeles-Olive \& Plco & CDMG & 34.04 & 118.26 & 193.4 & 187.1 & 0.022 & 6.7 & 5.4 \\
\hline 24752 & Los Angeles-Griffith Park/Toyon(Rock) & CDMG & 34.14 & 118.30 & 193.6 & 185.7 & 0.013 & 2.8 & 2.4 \\
\hline 24612 & Los Angeles-Pico \& Sentous & CDMG & 34.04 & 118.27 & 193.9 & 187.5 & 0.018 & 4.9 & 5.6 \\
\hline 24853 & Los Angeles-Beverly Blvd LADOT & CDMG & 34.08 & 118.29 & 194.1 & 187.1 & 0.025 & 4.6 & 3.9 \\
\hline 14826 & Los Angeles-52nd \& Central & CDMG & 34.00 & 118.25 & 194.3 & 188.5 & 0.025 & 8.1 & 7.1 \\
\hline 24976 & Los Angeles-I10/110 Interchange Grnds & CDMG & 34.04 & 118.28 & 194.4 & 188.1 & 0.020 & 5.2 & 5.5 \\
\hline 14868 & Long Beach-Willow \& Cherry & CDMG & 33.81 & 118.16 & 195.0 & 192.1 & 0.020 & 9.0 & 10.6 \\
\hline 24816 & Los Angeles-Vermont \& Pico & CDMG & 34.05 & 118.29 & 195.4 & 188.8 & 0.019 & 4.9 & 6.0 \\
\hline 14823 & Los Angeles-103rd \& Compton & CDMG & 33.94 & 118.25 & 195.6 & 190.5 & 0.032 & 10.5 & 8.9 \\
\hline 24811 & Los Angeles-Melrose \& Western & CDMG & 34.08 & 118.31 & 195.7 & 188.6 & 0.030 & 5.2 & 3.9 \\
\hline 24852 & Los Angeles-USC Fire Station & CDMG & 34.02 & 118.28 & 195.7 & 189.5 & 0.015 & 6.5 & 5.5 \\
\hline 24810 & Hollywood-Franklin \& Bronson & CDMG & 34.10 & 118.32 & 195.9 & 188.5 & 0.035 & 4.5 & 3.3 \\
\hline 14787 & Los Angeles-MLK Hospital Grounds & CDMG & 33.92 & 118.24 & 196.1 & 191.4 & 0.038 & 9.9 & 8.7 \\
\hline 24709 & Los Angeles-USC Grounds & CDMG & 34.02 & 118.29 & 196.2 & 190.0 & 0.017 & 5.8 & 5.6 \\
\hline 24088 & Pacoima-Kagel Canyon Fire Sta & CDMG & 34.30 & 118.38 & 196.2 & 186.1 & 0.032 & 5.5 & 4.7 \\
\hline 14403 & Los Angeles-116th St. School & CDMG & 33.93 & 118.26 & 197.4 & 192.5 & 0.028 & 8.6 & 8.1 \\
\hline 14871 & Long Beach-Ocean Blvd \& Cherry & CDMG & 33.76 & 118.17 & 197.6 & 195.2 & 0.025 & 13.1 & 11.6 \\
\hline 24271 & Lake Hughes \#1-Fire Station \#78 & CDMG & 34.67 & 118.43 & 198.3 & 183.5 & 0.053 & 5.6 & 1.9 \\
\hline 14847 & Long Beach-Santa Fe Ave \& I405 & CDMG & 33.83 & 118.22 & 198.3 & 194.9 & 0.023 & 11.9 & 10.7 \\
\hline 14821 & Los Angeles-Vermont \& 78th & CDMG & 33.97 & 118.29 & 198.4 & 192.8 & 0.030 & 7.6 & 8.1 \\
\hline 24865 & Universal City-Hwy 101 \& Lankershim & CDMG & 34.14 & 118.36 & 199.0 & 191.0 & 0.035 & 4.1 & 2.1 \\
\hline 24809 & Hollywood-Sunset \& Fairfax & CDMG & 34.10 & 118.35 & 199.2 & 191.8 & 0.019 & 3.6 & 3.1 \\
\hline 14560 & Long Beach-City Hall Grounds & CDMG & 33.77 & 118.20 & 199.6 & 197.0 & 0.017 & 13.2 & 12.9 \\
\hline 24851 & Los Angeles-3rd \& La Brea LADOT & CDMG & 34.07 & 118.35 & 199.6 & 192.5 & 0.021 & 5.2 & 4.2 \\
\hline 24815 & North Hollywood-Laurel Cyn \& Sherman & CDMG & 34.20 & 118.40 & 200.3 & 191.4 & 0.029 & 5.4 & 4.6 \\
\hline 24818 & Los Angeles-Washington \& La Brea & CDMG & 34.04 & 118.35 & 200.7 & 194.0 & 0.017 & 5.1 & 5.1 \\
\hline 14820 & Los Angeles-54th \& Crenshaw & CDMG & 33.99 & 118.34 & 201.9 & 195.8 & 0.024 & 7.1 & 6.3 \\
\hline 24763 & Sylmar-Olive View Hospital Grounds & CDMG & 34.33 & 118.44 & 201.9 & 191.3 & 0.030 & 5.9 & 4.4 \\
\hline 14824 & Los Angeles-Vermont \& 182nd & CDMG & 33.87 & 118.29 & 202.5 & 198.2 & 0.020 & 10.6 & 11.1 \\
\hline 24765 & Panorama City-Kaiser Hospital Grounds & CDMG & 34.22 & 118.43 & 202.7 & 193.5 & 0.044 & 7.0 & 5.9 \\
\hline 14537 & Inglewood-Hollywood Park & CDMG & 33.95 & 118.33 & 202.9 & 197.3 & 0.020 & 8.1 & 6.6 \\
\hline 24808 & Van Nuys-Burbank \& Fulton & CDMG & 34.17 & 118.42 & 203.1 & 194.5 & 0.028 & 5.9 & 4.2 \\
\hline 24087 & Arieta-Nordhoff Ave Fire Sta & CDMG & 34.24 & 118.44 & 203.2 & 193.8 & 0.025 & 6.1 & 6.2 \\
\hline 24801 & Studio City-Ventura \& Coldwater Cyn Av & CDMG & 34.15 & 118.41 & 203.2 & 194.9 & 0.036 & 5.3 & 3.0 \\
\hline 14834 & Hawthome-Crenshaw \& EI Segundo & CDMG & 33.92 & 118.33 & 203.4 & 198.2 & 0.022 & 7.1 & 7.5 \\
\hline 24703 & Los Angeles-I10/La Cienega Geo. Array & CDMG & 34.04 & 118.38 & 203.5 & 196.8 & 0.030 & 7.0 & 9.3 \\
\hline 14846 & Carson-Main \& Sepulveda & CDMG & 33.81 & 118.28 & 204.1 & 200.5 & 0.014 & 9.9 & 9.6 \\
\hline 14835 & Wilmington-Avalon \& Anaheim & CDMG & 33.78 & 118.26 & 204.3 & 201.2 & 0.017 & 9.7 & 9.8 \\
\hline 24800 & Mission Hills-Sepulveda \& San Fernando & CDMG & 34.27 & 118.46 & 204.7 & 194.7 & 0.028 & 7.9 & 7.3 \\
\hline 24867 & Van Nuys-Civic Center Grounds & CDMG & 34.18 & 118.45 & 205.2 & 196.4 & 0.026 & 7.0 & 4.4 \\
\hline 14767 & Los Angeles-LAPD Manchester Cntr Grnds & CDMG & 33.96 & 118.38 & 206.2 & 200.4 & 0.017 & 9.9 & 6.9 \\
\hline 14785 & Los Angeles-Vincent Thos. E Geo. Array & CDMG & 33.75 & 118.27 & 206.6 & 203.8 & 0.024 & 12.3 & 10.0 \\
\hline 14845 & Harbor City-Normandle \& Pac. Coast Hwy & CDMG & 33.79 & 118.30 & 206.8 & 203.3 & 0.014 & 9.8 & 8.9 \\
\hline 14822 & Los Angeles-Century \& Aviation & CDMG & 33.94 & 118.38 & 207.1 & 201.4 & 0.017 & 7.4 & 7.4 \\
\hline 14786 & Los Angeles-Vincent Thos. W Geo. Array & CDMG & 33.75 & 118.28 & 207.4 & 204.5 & 0.029 & 10.7 & 8.1 \\
\hline JFP & Jensen Filtration & SCSN & 34.31 & 118.50 & 207.5 & 197.0 & 0.046 & 7.1 & 5.0 \\
\hline 14831 & Lomita-Narbonne \& Pacific Coast Hwy & CDMG & 33.79 & 118.32 & 208.6 & 205.0 & 0.015 & 9.1 & 8.3 \\
\hline 24909 & Los Angeles-I10/405 Freefield & CDMG & 34.03 & 118.43 & 208.6 & 201.7 & 0.023 & 5.2 & 5.5 \\
\hline 24819 & Los Angeles-Veteran \& Wilshire & CDMG & 34.06 & 118.45 & 208.9 & 201.5 & 0.017 & 3.8 & 2.7 \\
\hline 24279 & Newhall-County Fire Sta. & CDMG & 34.39 & 118.53 & 209.1 & 197.6 & 0.021 & 4.5 & 4.3 \\
\hline
\end{tabular}


Table 1

Continued

\begin{tabular}{|c|c|c|c|c|c|c|c|c|c|}
\hline $\begin{array}{l}\text { Station } \\
\text { ID }\end{array}$ & Station Name & Network & Lat & Long & $\begin{array}{c}\text { Epi Dist } \\
(\mathrm{km})\end{array}$ & $\begin{array}{l}\text { Fault Dist } \\
(\mathrm{km})\end{array}$ & $\begin{array}{c}\text { Accel } \\
(\mathrm{g})\end{array}$ & $\begin{array}{c}\text { Vel } \\
(\mathrm{cm} / \mathrm{sec})\end{array}$ & $\begin{array}{l}\text { Displ } \\
(\mathrm{cm})\end{array}$ \\
\hline 24586 & Neenach-Sacatara Creek & CDMG & 34.85 & 118.54 & 209.5 & 192.9 & 0.023 & 5.7 & 3.5 \\
\hline 24802 & Encino-Balboa \& Ventura & CDMG & 34.16 & 118.50 & 210.5 & 201.8 & 0.021 & 2.8 & 2.3 \\
\hline 14159 & San Pedro-Palos Verdes Fire Station & CDMG & 33.72 & 118.31 & 211.2 & 208.5 & 0.008 & 4.0 & 3.2 \\
\hline 24805 & Northridge-Parthenia \& Lindley & CDMG & 34.23 & 118.53 & 211.3 & 201.7 & 0.039 & 10.5 & 6.9 \\
\hline 24817 & Los Angeles-Sunset \& Kenter & CDMG & 34.06 & 118.48 & 211.7 & 204.2 & 0.012 & 3.2 & 3.5 \\
\hline MIS & Mira Catallna School & SCSN & 33.74 & 118.33 & 212.5 & 209.4 & 0.012 & 4.0 & 3.1 \\
\hline $24 \mathrm{~T} 03$ & Tarzana-Clubhouse & CDMG & 34.16 & 118.53 & 213.5 & 204.6 & 0.029 & 3.5 & 2.3 \\
\hline 24764 & Tarzana-Cedar Hill B & CDMG & 34.16 & 118.53 & 213.6 & 204.8 & 0.055 & 5.1 & 2.1 \\
\hline 24866 & Reseda-Vanowen \& Wilbur LADOT & CDMG & 34.19 & 118.55 & 214.0 & 204.7 & 0.031 & 9.6 & 5.3 \\
\hline NOT & Northridge & SCSN & 34.23 & 118.56 & 214.2 & 204.5 & 0.033 & 8.9 & 9.3 \\
\hline 24806 & Canoga Park-Winnetka \& Roscoe & CDMG & 34.22 & 118.57 & 215.4 & 205.7 & 0.032 & 9.7 & 6.4 \\
\hline 1794 & EI Centro-Meloland Geotechnical Array & CDMG & 32.77 & 115.45 & 215.5 & 188.1 & 0.017 & 9.5 & 6.9 \\
\hline 24278 & Castaic-Old Ridge Route & CDMG & 34.56 & 118.64 & 217.7 & 204.0 & 0.044 & 5.1 & 2.1 \\
\hline 24277 & Castaic-Hasley Canyon & CDMG & 34.46 & 118.65 & 219.0 & 206.5 & 0.022 & 4.4 & 3.0 \\
\hline 24807 & Woodland Hills-Canoga \& Ventura & CDMG & 34.17 & 118.60 & 219.0 & 209.9 & 0.030 & 4.5 & 3.0 \\
\hline 3748 & San Diego-UCSD Hospital Grounds & CDMG & 32.75 & 117.16 & 219.9 & 162.5 & 0.010 & 3.1 & 1.6 \\
\hline 24804 & West Hills-Roscoe \& Fallbrook & CDMG & 34.22 & 118.63 & 220.6 & 210.8 & 0.037 & 6.4 & 3.2 \\
\hline 24803 & Woodland Hills-Victory \& Fallbrook & CDMG & 34.19 & 118.62 & 221.0 & 211.7 & 0.028 & 5.6 & 3.6 \\
\hline 24861 & Simi Valley-Katherine Rd \& Sylvan & CDMG & 34.26 & 118.67 & 223.5 & 213.2 & 0.012 & 3.7 & 3.0 \\
\hline 24644 & Sandberg-Bald Mountain & CDMG & 34.74 & 118.72 & 225.6 & 210.1 & 0.019 & 3.0 & 1.9 \\
\hline 24860 & Simi Valley-Church St \& Los Angeles Av & CDMG & 34.27 & 118.74 & 229.3 & 215.8 & 0.021 & 4.3 & 3.4 \\
\hline SMV & Simi Valley & SCSN & 34.27 & 118.74 & 230.1 & 219.6 & 0.020 & 4.2 & 3.4 \\
\hline 24855 & Piru-Church St \& Camulos & CDMG & 34.41 & 118.80 & 232.8 & 220.6 & 0.031 & 6.6 & 3.4 \\
\hline 24859 & Oak Park-Kanan \& Deerhill & CDMG & 34.18 & 118.76 & 233.5 & 223.8 & 0.013 & 2.4 & 2.2 \\
\hline 24396 & Mallbu-Point Dume & CDMG & 34.01 & 118.80 & 241.5 & 233.6 & 0.015 & 3.4 & 3.1 \\
\hline 24283 & Moorpark-Fire Station & CDMG & 34.29 & 118.88 & 242.3 & 231.3 & 0.016 & 4.6 & 4.7 \\
\hline 24864 & Somis-Somis Rd & CDMG & 34.26 & 119.00 & 253.2 & 242.3 & 0.016 & 3.6 & 2.7 \\
\hline 24863 & Camarillo-Woodcreek \& Santa Rosa & CDMG & 34.22 & 118.99 & 253.7 & 243.2 & 0.017 & 4.6 & 2.8 \\
\hline 25282 & Camarillo & CDMG & 34.21 & 119.08 & 261.7 & 251.2 & 0.017 & 4.7 & 3.1 \\
\hline 35365 & Bakersfield-Stockdale \& California & CDMG & 35.35 & 119.05 & 268.0 & 248.0 & 0.008 & 2.9 & 3.6 \\
\hline 25862 & Oxnard-Hwy 101 \& Oxnard Blvd & CDMG & 34.24 & 119.18 & 270.2 & 259.2 & 0.014 & 7.8 & 6.6 \\
\hline 25854 & Ojai-Ojai Av \& Fairway Ln & CDMG & 34.45 & 119.23 & 272.2 & 259.3 & 0.013 & 2.7 & 1.9 \\
\hline 44015 & Lone Pine A & CDMG & 36.60 & 118.06 & 275.9 & 251.1 & 0.015 & 4.3 & 3.5 \\
\hline
\end{tabular}

$(216 \mathrm{~km})$ display an extremely-long duration $(100-120 \mathrm{sec})$ of long-period $(4-8 \mathrm{sec})$ motions. Surface waves generated in the deep sedimentary basins along the propagation path may be responsible for these effects.

In general, two types of ground displacement are observed during the Hector Mine earthquake:

1. One is a relatively simple motion with a duration of 15 $20 \mathrm{sec}$. This type of motion can be observed everywhere, from $50 \mathrm{~km}$ up to $240 \mathrm{~km}$ from the epicenter. This motion may be associated with the source-generated $S$ wave.

2. Motion of the second type is much longer in duration, about $40 \mathrm{sec}$; this type of motion is sinusoidal with a predominant period of about 5 to $8 \mathrm{sec}$. This type of motion is typical for certain locations in the Los Angeles, Long Beach, San Bernardino, and EI Centro areas. This displacement may be relatively high amplitude (more than $10 \mathrm{~cm}$ at the epicentral distances of about $200 \mathrm{~km}$ ). The amplitude of this motion increases with time and reaches a maximum approximately $20 \mathrm{sec}$ after the arrival of the $S$ wave. This sinusoidal type of motion is most likely Love waves created by multiple reflections of $S$ waves trapped in a deep sedimentary basin.

The N-S components of displacement waveforms recorded by 50 representative TriNet stations are shown on a map of southern California along with Quaternary, Tertiary, and Mesozoic (QTM) geology (Jennings, 1977; Park and Elrick, 1998) (Fig. 3). Surface fault rupture produced by the Hector Mine earthquake is shown in red in Figure 3 (Treiman et al., 2002). The map shows the distribution of displacement waveforms relative to the fault and QTM geology (Jennings, 1994). Since the Hector Mine earthquake occurred in the Mojave Desert area, not many strong-motion stations that recorded the earthquake are located within 100 $\mathrm{km}$ of the epicenter. High-displacement amplitudes with long duration of ground motion occurred in the Los Angeles and Long Beach areas at the epicentral distance of about 200 $\mathrm{km}$. Seismic waves with high amplitudes and long duration at periods of 5-8 sec may have significant effects on large structures.

In general, no direct correlation can be seen between 


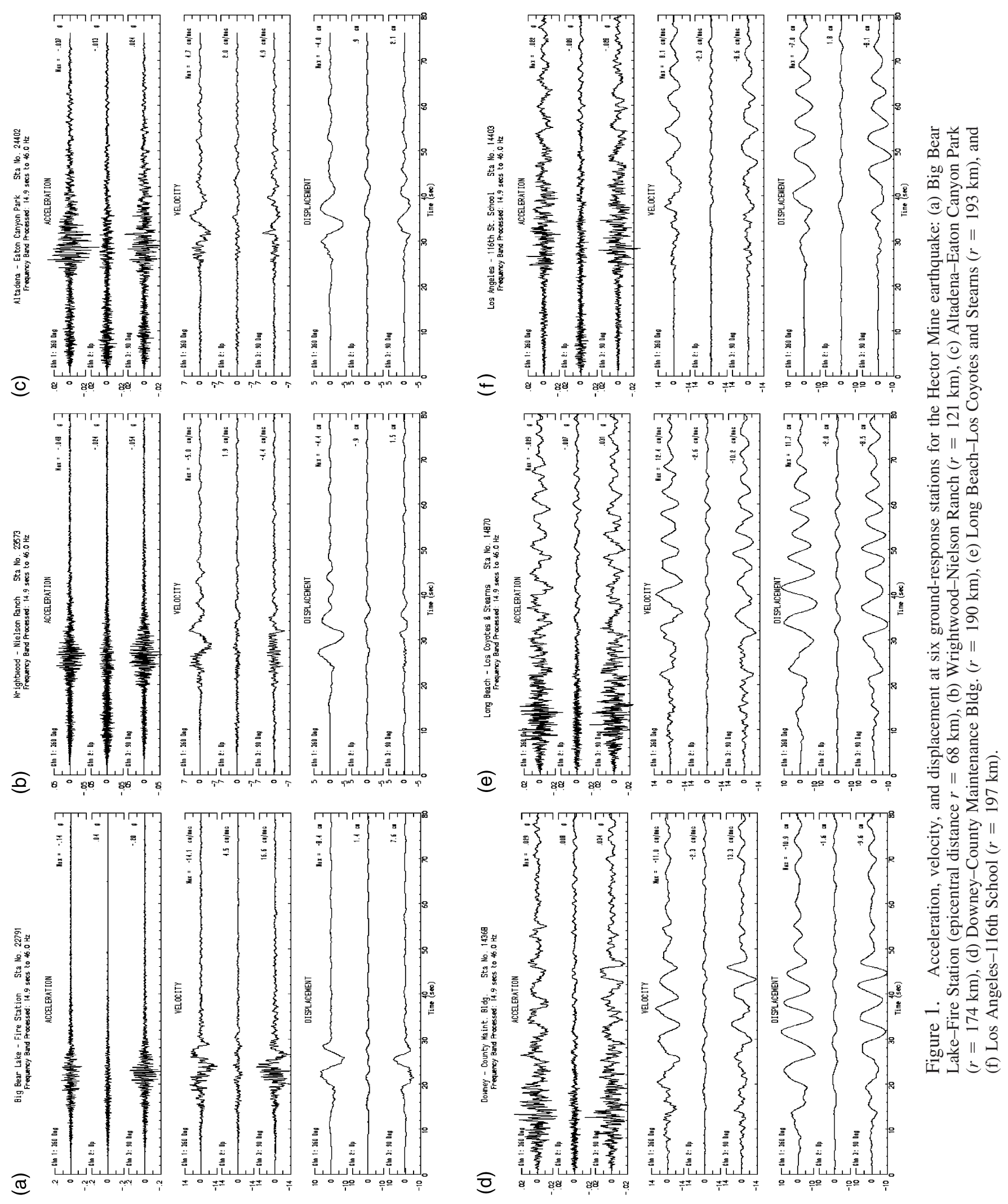


Table 2

Peak Horizontal Ground Motions for the Records Shown in Figure 1

\begin{tabular}{llccrr}
\hline $\begin{array}{l}\text { Station } \\
\text { ID }\end{array}$ & \multicolumn{1}{c}{ Station Name } & $\begin{array}{c}\text { Epi Dist } \\
(\mathrm{km})\end{array}$ & $\begin{array}{c}\text { PGA } \\
(\mathrm{g})\end{array}$ & $\begin{array}{c}\text { PGV } \\
(\mathrm{cm} / \mathrm{sec})\end{array}$ & $\begin{array}{r}\text { PGD } \\
(\mathrm{cm})\end{array}$ \\
\hline 22791 & Big Bear Lake-Fire Station & 68 & 0.20 & 16.6 & 8.4 \\
23573 & Wrightwood-Nielson Ranch & 121 & 0.054 & 5.0 & 4.4 \\
24402 & Altadena-Eaton Canyon Park & 174 & 0.037 & 4.9 & 4.0 \\
14368 & Downey-County Maintenance Bldg & 190 & 0.034 & 13.3 & 10.9 \\
14870 & Long Beach-Los Coyotes \& Stearns & 193 & 0.031 & 12.4 & 11.7 \\
14403 & Los Angeles-116th St. School & 197 & 0.028 & 8.6 & 8.1 \\
\hline
\end{tabular}

Comparison of $5 \%$ damped spectra

(a)

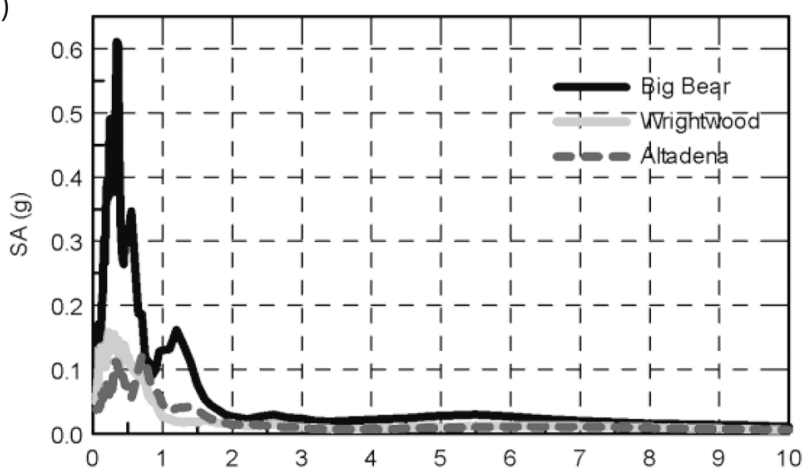

(b)

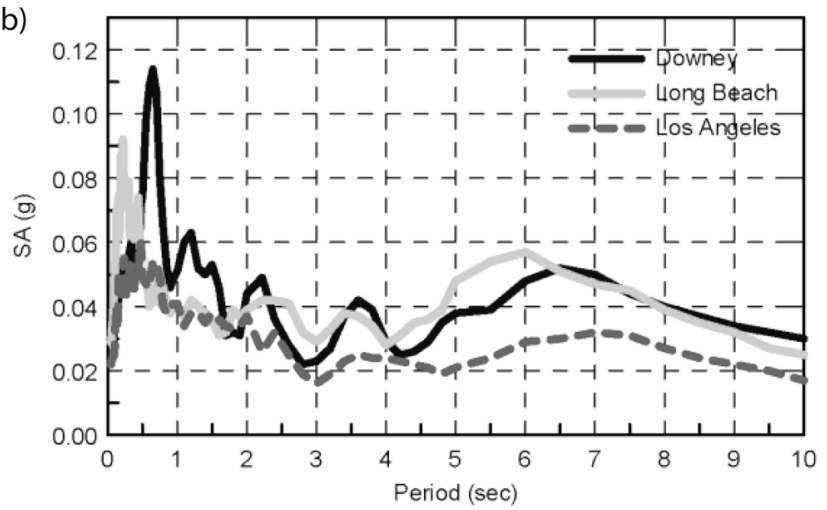

Figure 2. Response spectra (5\% damped) of the $\mathrm{N}-\mathrm{S}$ components for the six stations shown in Fig. 1: (a) for Big Bear, Wrightwood, and Altadena; (b) for Downey, Long Beach, and Los Angeles.

QTM geology and ground displacements. Figure 3 graphically shows large variations in displacements, as seen in some areas near the ocean, where both types of motion (the simple 15- to 20-sec long and the sinusoidal 40 -sec long) were recorded at nearby stations. In this specific set of data, directivity does not appear to play a significant role; however, the spatial distribution of the strong-motion stations, located mostly to the west and south of the earthquake fault at epicentral distances of more than $80 \mathrm{~km}$, is not favorable for studying directivity effects.

\section{Response of Tarzana}

Significantly amplified ground accelerations at the Tarzana station were recorded during the $M 5.9$ Whittier Narrows earthquake and during the $M 6.7$ Northridge earthquake and its aftershocks (Shakal et al., 1988, 1996; Darragh et al., 1997, 1998). In contrast, the Landers, Big Bear, and Sierra Madre mainshocks, and some other earthquakes, produced lower site amplifications. Amplified seismic response was observed at the Tarzana station during the Hector Mine earthquake (at an epicentral distance of $214 \mathrm{~km}$ ), with peak horizontal ground acceleration almost twice as large as the accelerations recorded at other stations located at the same epicentral distance (Table 1).

The two strong-motion stations located at TarzanaCedar Hill B (having a three-component instrument at the surface and another one downhole at the depth of $60 \mathrm{~m}$ ) and Clubhouse (about $180 \mathrm{~m}$ from Cedar Hill B, at the foot of Tarzana hill) have again demonstrated significant differences in maximum amplitudes of acceleration ( 0.055 and $0.036 \mathrm{~g}$, respectively) and velocity (5.0 and $3.5 \mathrm{~cm} / \mathrm{sec}$ ), with almost no difference in maximum displacements $(2.5$ and $2.4 \mathrm{~cm}$ ). Figure 4 shows acceleration, velocity, and displacement time histories recorded at the downhole and two different surface locations. Ground motions were rotated to the directions parallel and perpendicular to the strike of the Tarzana hill.

Comparison of motions at the bottom and top of the hole shows significant amplification of accelerations (more than three times for the component perpendicular to the hill). In contrast to accelerations (high-frequency part of the seismic signal), displacements (relatively low-frequency part of the signal) demonstrate almost no site amplification from the bottom of the hole to the surface at long periods. Ground displacements at other CSMIP-instrumented downhole sites that recorded the Hector Mine earthquake also demonstrate almost no near-surface site amplification at longer periods (Graizer et al., 2000a,b).

The downhole data are used as a reference to compare the amplification effect at the top of Tarzana hill (Hill B) and at the foothill (Clubhouse). Amplification from the bottom of the hole to the surface is similar along the component parallel to the strike of Tarzana hill to that at the foothill station, but the amplification from the bottom of the hole to 


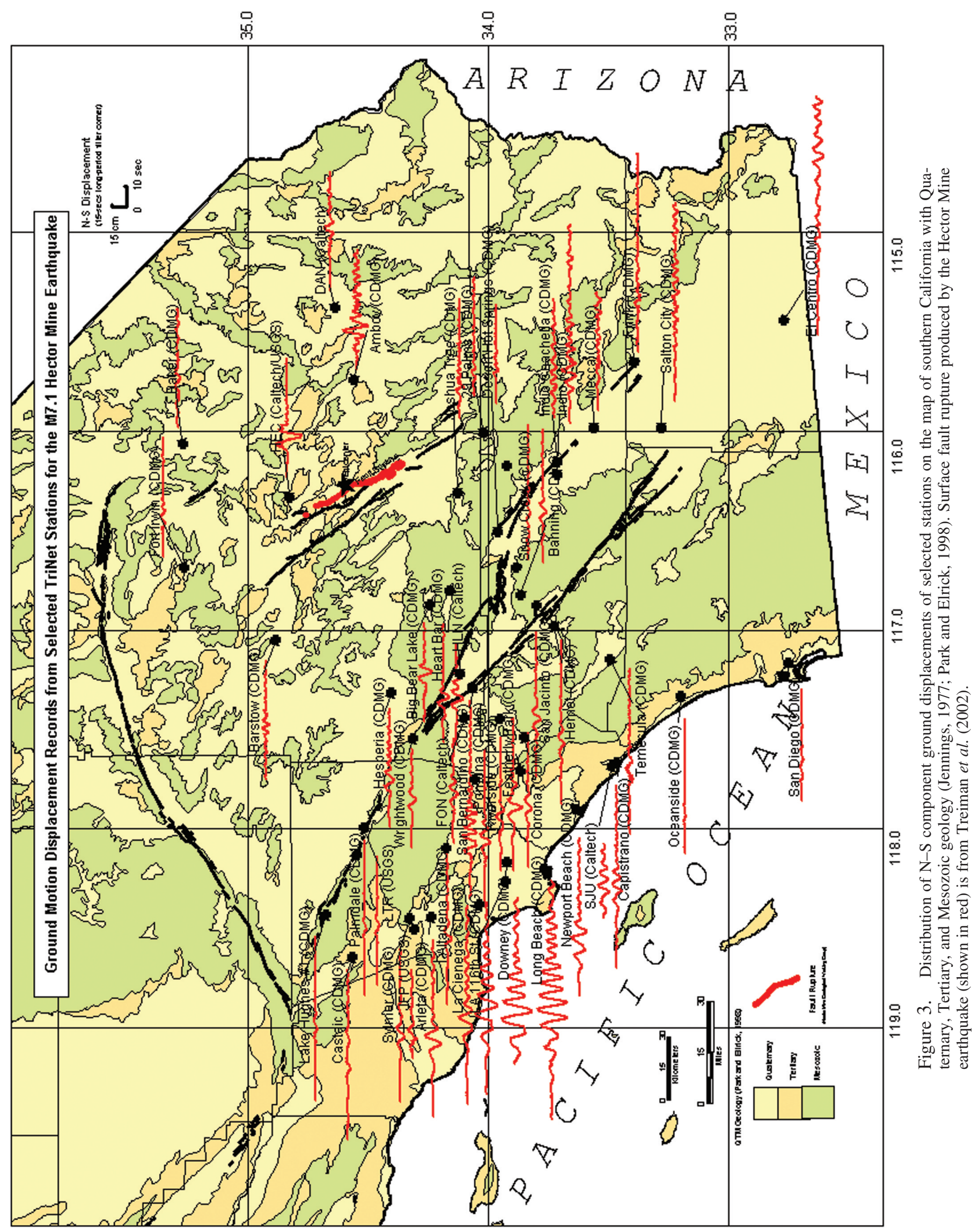




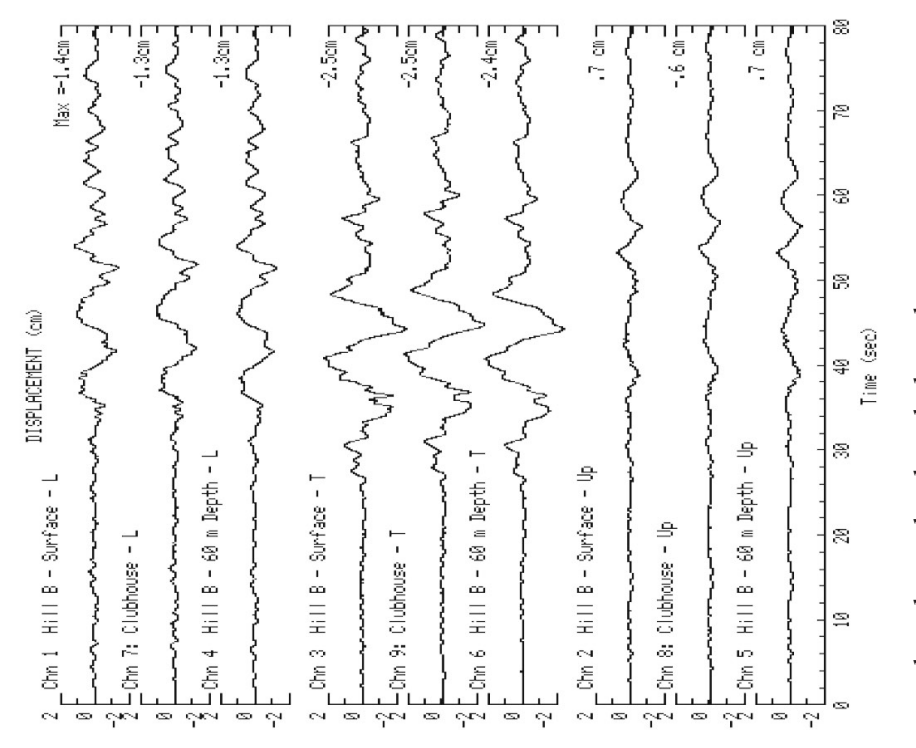

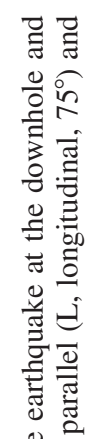

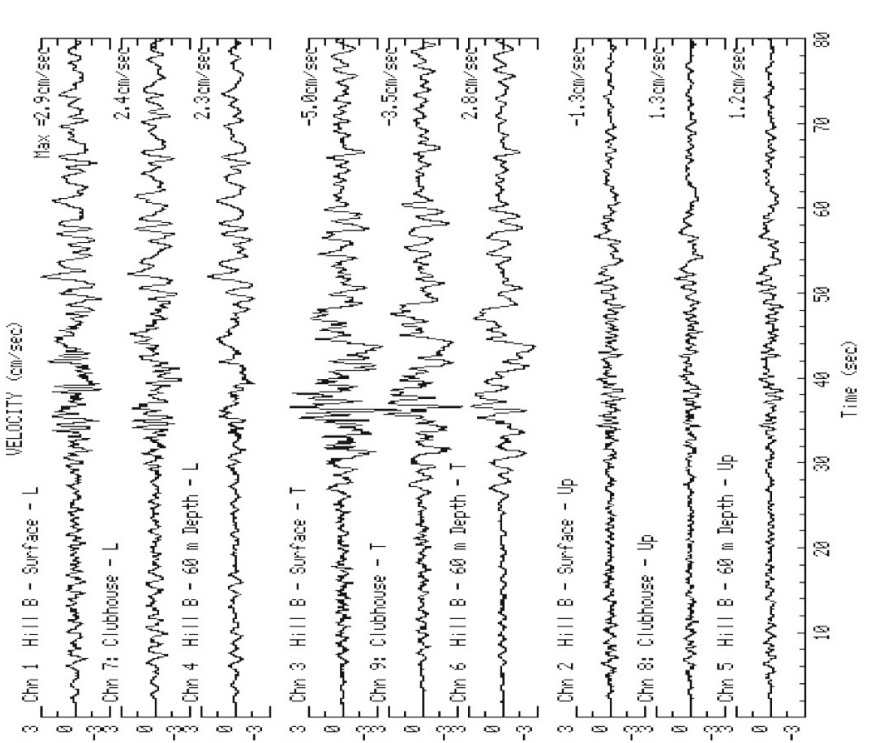

帘芯 氝: ․․ bo 吾 急 흘 임 䒕节家 过它 ֻू. 可品 응

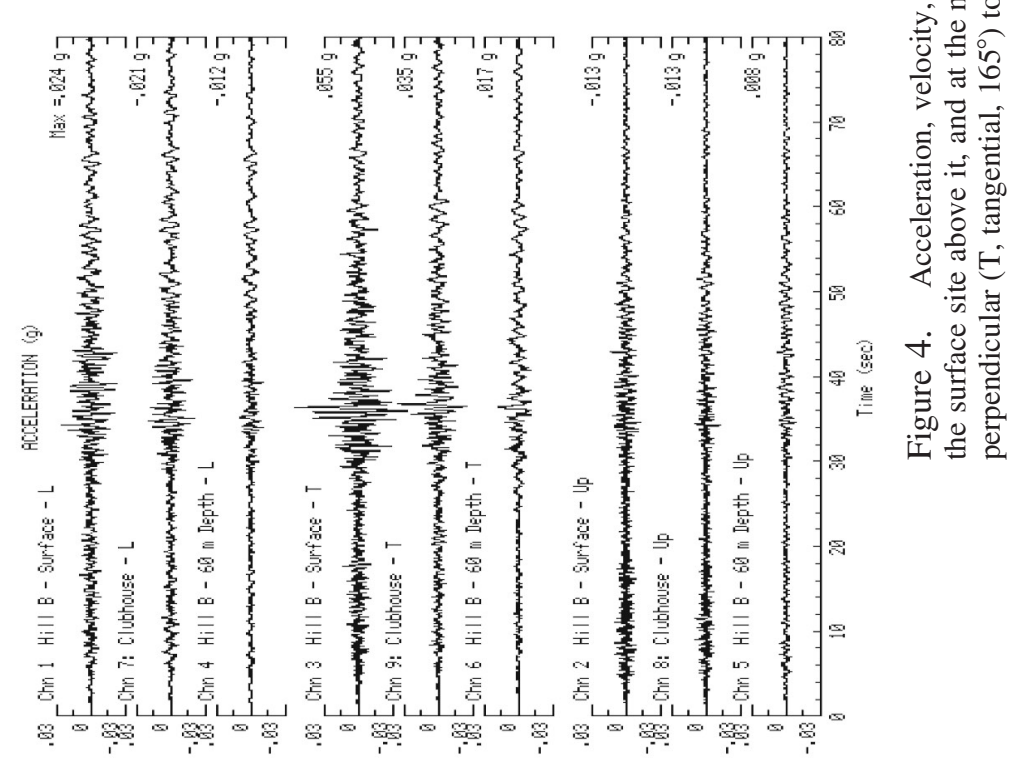


(a)

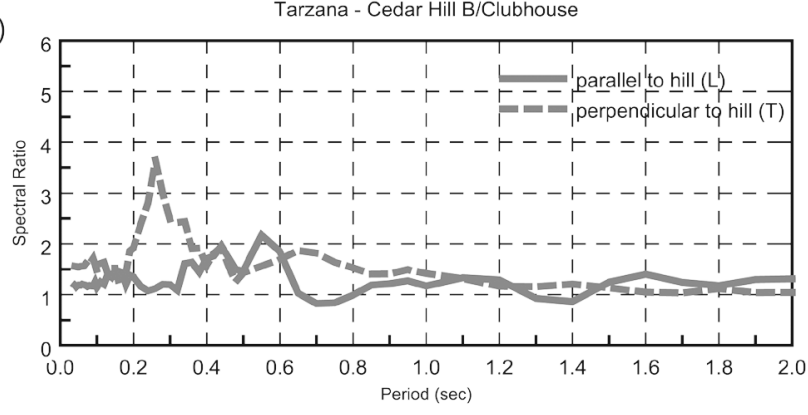

(b)

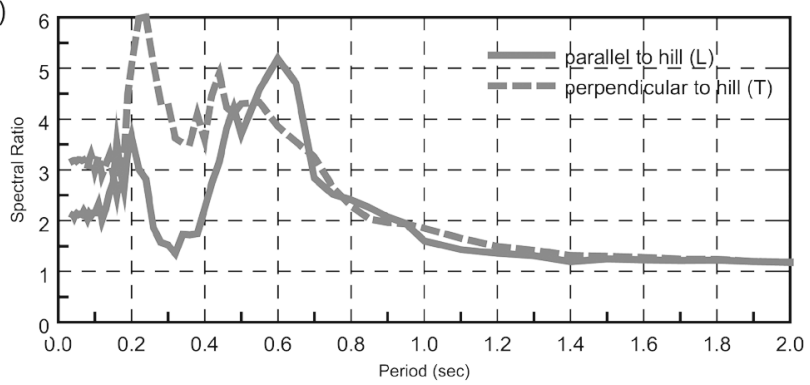

Clubhouse/60 $\mathrm{m}$ depth

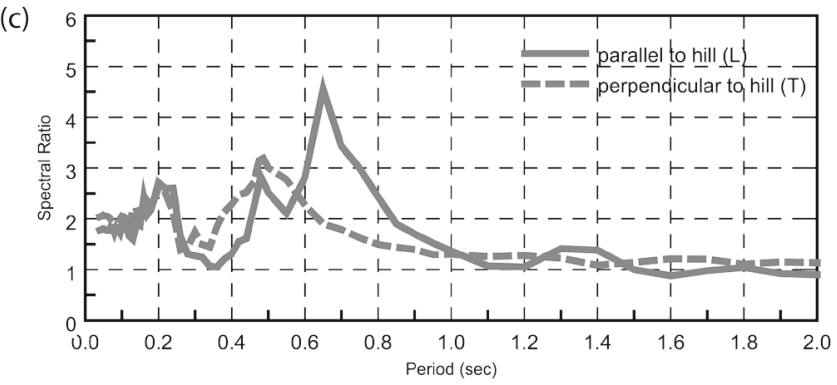

Figure 5. Spectral ratios of the response spectra (5\% damped) at Tarzana. Ratios of (a) Cedar Hill B to Clubhouse, (b) Cedar Hill B to $60 \mathrm{~m}$ depth, (c) and Clubhouse to $60 \mathrm{~m}$ depth for the component parallel to the hill (solid line) and perpendicular to the hill (dashed line).

the surface is more than double along the component perpendicular to the strike of the hill at a period of $0.22 \mathrm{sec}$ (4.5 Hz) (Fig. 5).

Comparison of the response spectra demonstrates clear directional site response resonance at Tarzana hill, with an amplification of more than 3.5 between the foothill station and the top of the hill at a period of $0.3 \mathrm{sec}$ along the component perpendicular to the hill (Fig. 5). This result is similar to previously obtained results from aftershocks of the Northridge earthquake (Vidale et al., 1991; Hartzell et al., 1996; Spudich et al., 1996; Darragh et al., 1998). The threedimensional topographic effect (Bouchon and Barker, 1996; Catchings and Lee, 1996; Spudich et al., 1996) only partially explains the site amplification on the top of the hill.

\section{Ground Motion Attenuation}

Plots of the Hector Mine peak horizontal ground acceleration (PGA), velocity (PGV), and displacement (PGD) re- corded by the ground-response stations listed in Table 1 are shown in Figure 6. The PGA, PGV, and PGD demonstrate the differences in amplitude attenuation of different parts of the seismic spectra in the epicentral distance range of 30 to $280 \mathrm{~km}$.

Peak ground acceleration (Fig. 6a) represents the relatively high-frequency part of seismic radiation. It is associated with wave periods of up to $1 \mathrm{sec}$ (frequencies higher than $1 \mathrm{~Hz}$ ). PGA decreases with increasing epicentral distance to about $150 \mathrm{~km}$, but stays relatively constant at greater distances.

Peak ground velocity (Fig. 6b), representing the midrange frequencies of 0.1 to $3 \mathrm{~Hz}$ (periods of $0.3-10 \mathrm{sec}$ ), also demonstrates attenuation, but with much more variability. PGV generally follows the same pattern as PGA, decreasing with epicentral distance and reaching a level of approximately 3 to $5 \mathrm{~cm} / \mathrm{sec}$ at a distance of about $220 \mathrm{~km}$. It also demonstrates more variability than PGA, especially at epicentral distances of 120 to $220 \mathrm{~km}$.

Peak ground displacement (Fig. 6c), which is mostly associated with the relatively long-period part of the spectrum (generally, $2 \mathrm{sec}$ and longer; frequencies less than 0.5 $\mathrm{Hz}$ ), shows very high variability and little attenuation with distance, with anomalous amplification at about 160 to 210 $\mathrm{km}$ distance from the epicenter. The increased amplification may reflect the shift from motions associated with sourcegenerated $S$ waves to the basin-generated or surface waves. PGD reaches a level of approximately 2 to $4 \mathrm{~cm}$ at distances greater than $220 \mathrm{~km}$.

Variations in PGA data also are plotted with respect to the closest distance to the fault (in log-log scale) to allow comparison with the Boore-Joyner-Fumal (BJF) (Boore et al., 1993, 1997) relationship between PGA and fault distance. Coefficients for a strike-slip fault and average shearwave velocity of $520 \mathrm{~m} / \mathrm{sec}$ in the upper $30 \mathrm{~m}$ are used to calculate the empirical prediction BJF97 curve (Fig. 7). This velocity is recommended for use in BJF97 for the National Earthquake Hazards Reduction Program (NEHRP) site class C. The original BJF97 relationship for peak horizontal accelerations is

$$
\operatorname{In} Y=b_{1}+b_{2}(M-6)+b_{5} \operatorname{In} r+b_{\mathrm{v}} \operatorname{In}\left(V_{\mathrm{S}} / V_{\mathrm{A}}\right),
$$

where

$$
\left.r=\sqrt{(} r_{\mathrm{jb}}{ }^{2}+h^{2}\right)
$$

$b_{1}=0.313$ (for strike-slip earthquakes)

$b_{2}=0.527, b_{5}=0.778$

$b_{\mathrm{v}}=0.371, V_{\mathrm{A}}=1396 \mathrm{~m} / \mathrm{sec}, h=5.57$.

Here $M$ is moment magnitude, $r_{\mathrm{jb}}$ is the closest horizontal distance (in $\mathrm{km}$ ) from the station to the point on the earth's surface that lies directly above the rupture, and $V_{\mathrm{S}}$ is the average shear-wave velocity in the upper $30 \mathrm{~m}$. The equation is limited to distances of $80 \mathrm{~km}$, because of lack of 

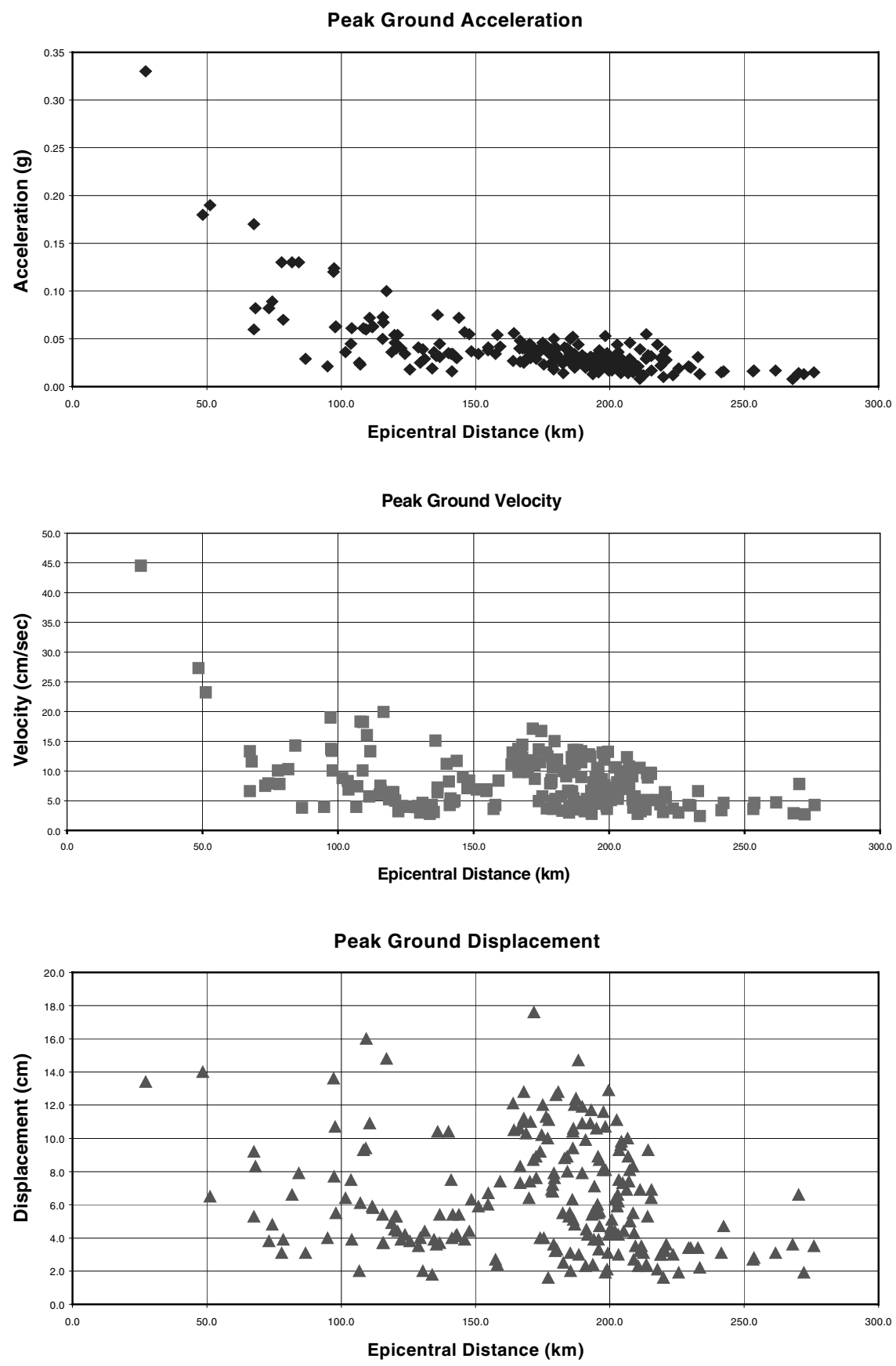

Figure 6. Hector Mine peak ground acceleration (PGA), velocity (PGV), and displacement (PGD) in the epicentral distance range of 30-280 km.

reliable data at greater distances, and to magnitudes $5.5<$ $M<7.5$. Peak ground accelerations from the Hector Mine earthquake demonstrate reasonable agreement with the BJF97 attenuation curve in the applicable range and possibly may be used to extend the attenuation relationships to greater distances.

Variations in acceleration, velocity, and displacement dependent upon the azimuth from the epicenter are demonstrated in the record section in Figure 8. Three record sections for the $\mathrm{N}-\mathrm{S}$ component of ground motion were con- structed at azimuths of approximately $255^{\circ}, 235^{\circ}$, and $215^{\circ}$ from the epicenter. To make comparison of the records more convenient, they were shifted so that the maximum phase in displacement of the $S$-wave arrivals are aligned. Accelerations in Figure 8 may be characterized as relatively long wavetrains with varying periods and amplitudes. Velocities also can be characterized by large variations in amplitudes and periods of motions.

Displacements in the $255^{\circ}$ azimuth in Figure $8 \mathrm{a}$ are similar in amplitude over a large range of epicentral dis- 


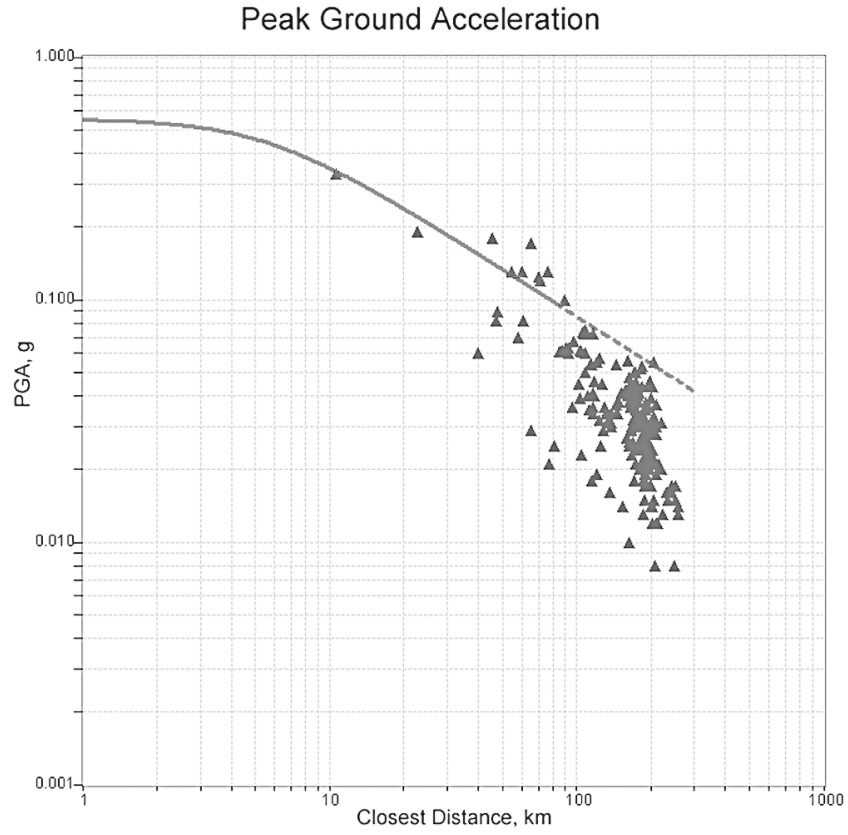

Figure 7. Peak horizontal ground acceleration values plotted against the closest distance to the fault, and the Boore-Joyner-Fumal (Boore et al., 1997) attenuation relationship. For comparison, extrapolation of the BJF97 relationship is shown with dashed line for distances beyond the original distance limitation presented by the authors.

tances, from 100 to $200 \mathrm{~km}$. Displacement records in the azimuth of $215^{\circ}$ (Fig. 8c) also demonstrate similarity in shape, but the amplitudes generally decrease with increasing epicentral distance. Displacements at alluvium sites on an azimuth crossing the Los Angeles basin $\left(235^{\circ}\right)$ have the biggest variations in wave shape and duration of oscillation (Fig. 8b). Amplitudes of the motion decrease significantly in the intermediate part of the profile at epicentral distances of 130 to $140 \mathrm{~km}$ (e.g., Riverside, Corona) and increase again at distances of 180 to $190 \mathrm{~km}$ (e.g., Newport Beach, Huntington Beach).

Large variations in ground motions may be due to the wave-propagation path and near-surface site geology (Tarzana and Temecula) or to the thickness and structure of the sedimentary basin near the station (e.g., San Bernardino, Los Angeles, and Long Beach). For example, ground motion at Temecula (Fig. 8c) has a relatively strong component with a period of $1.4 \mathrm{sec}(0.7 \mathrm{~Hz})$, which may be due to the relatively soft surface layer.

\section{Comparison with Landers Earthquake}

In contrast to the 1994 Northridge earthquake $\left(M_{\mathrm{w}} 6.7\right)$, the Hector Mine event $\left(M_{\mathrm{w}} 7.1\right)$ occurred in a desert area relatively far from Los Angeles. From the point of view of its magnitude and location relative to the main urban areas, this earthquake was more similar to the 1992 Landers earthquake $\left(M_{\mathrm{w}} 7.3\right)$.
The Landers earthquake was recorded by many strongmotion stations (e.g., Shakal et al., 1992; Cramer and Darragh, 1994), but many of them were film strong-motion accelerograph (SMA) instruments. Relatively few of the Hector Mine earthquake records were recorded by the film instruments, with most of the data being recorded by modern, high-quality digital accelerographs.

A comparison was made of strong ground motions recorded at six stations in the Los Angeles area during the Hector Mine earthquake (epicentral distances of about 190 $\mathrm{km}$ ) and the Landers earthquake of 1992 (epicentral distances of about $165 \mathrm{~km}$ ).

For comparison with the Landers earthquake, records at the following stations are studied: Los Angeles-City Terrace, Los Angeles-University Hospital Grounds, Los Angeles-116th street School, Los Angeles-Pico and Sentous, Los Angeles-Temple and Hope, and Los Angeles-Obregon Park. The records of the Landers earthquake used for comparison are reprocessed using the same frequency band 0.067 to $46 \mathrm{~Hz}(0.022-15 \mathrm{sec})$ as all the records from the Hector Mine earthquake. The peak horizontal ground accelerations recorded at these six stations are listed in Table 3, and Figure 9 is a comparison of the ground motions at two of the six stations. On average, peak ground accelerations during the Landers earthquake were 1.6 times larger than those of the Hector Mine event.

The response spectra from the 1992 Landers earthquake are higher than for the Hector Mine event, except at periods of 6 to $8 \mathrm{sec}$, where they are about the same value (Fig. 10). As we have suggested, these periods are most likely associated with the motion in the source of the Hector Mine earthquake. In the 1.5- to 3.0-sec period range, the Landers response spectra are two to three times higher than those of the Hector Mine earthquake. The amplification factor in PGA that one can expect from the difference in magnitude and distance for these two earthquakes using the BJF97 empirical prediction of ground motion gives a factor of 1.24 (Landers PGA/Hector PGA ratio, Table 3). Since the actual peak ground accelerations in the Los Angeles area recorded during the Landers earthquake were 1.6 times larger than the motions during the Hector Mine earthquake, not only differences in location and magnitude are responsible for this effect. Differences in rupturing and directivity of the source processes might also contribute to this amplification effect.

The Landers records are characterized not only by higher amplitudes, but also by longer durations of motion. The longer duration may be explained by the longer rupture length of the earthquake source. Comparison of the surface ruptures associated with the 1992 Landers and the $1999 \mathrm{Hec}-$ tor Mine earthquakes shows that the overall length of the first event was approximately 1.8 time larger (Scientists of the USGS et al., 2000). Using source modeling, the Landers fault length was estimated to be $65 \mathrm{~km}$ (Wald and Heaton, 1994) and the length of the Hector Mine source was estimated to be approximately $35 \mathrm{~km}$ (Dreger and Kaverina, 2000a,b). 

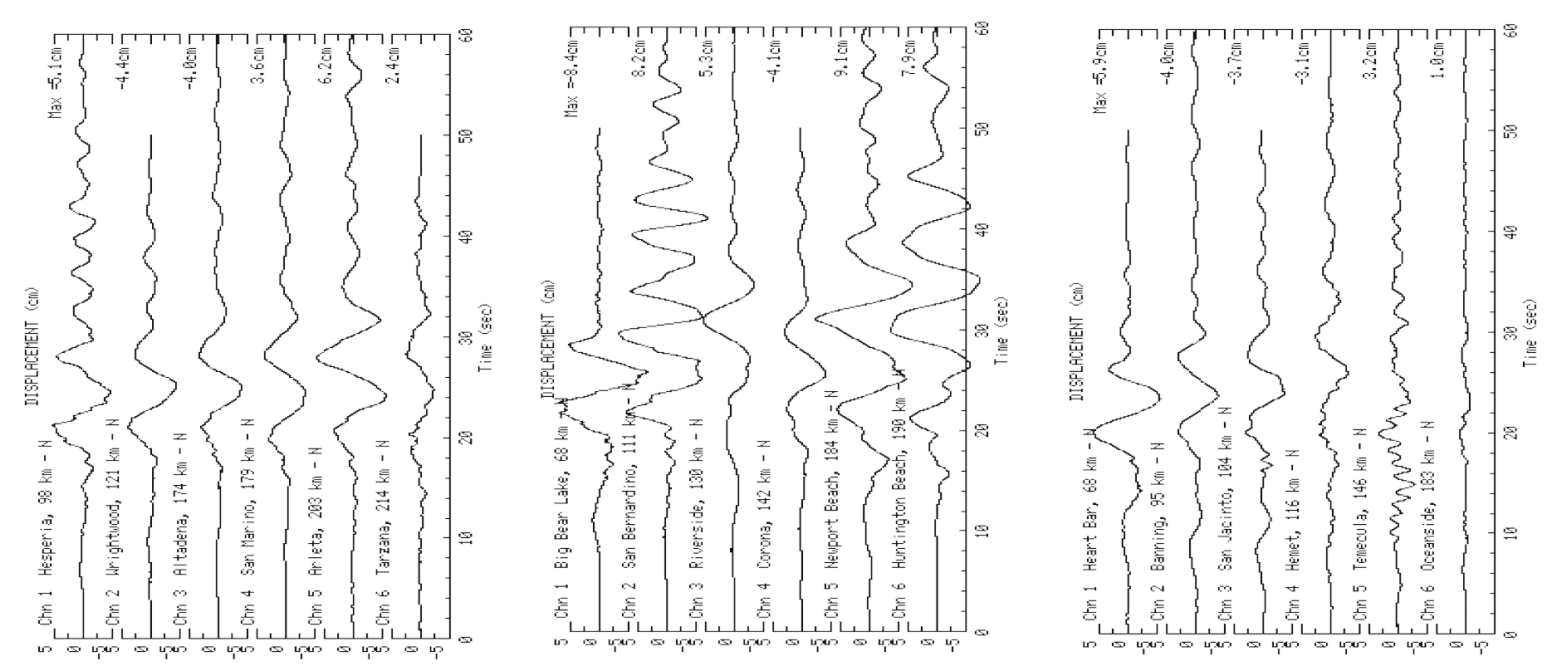

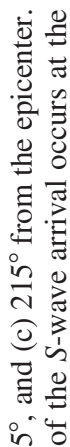
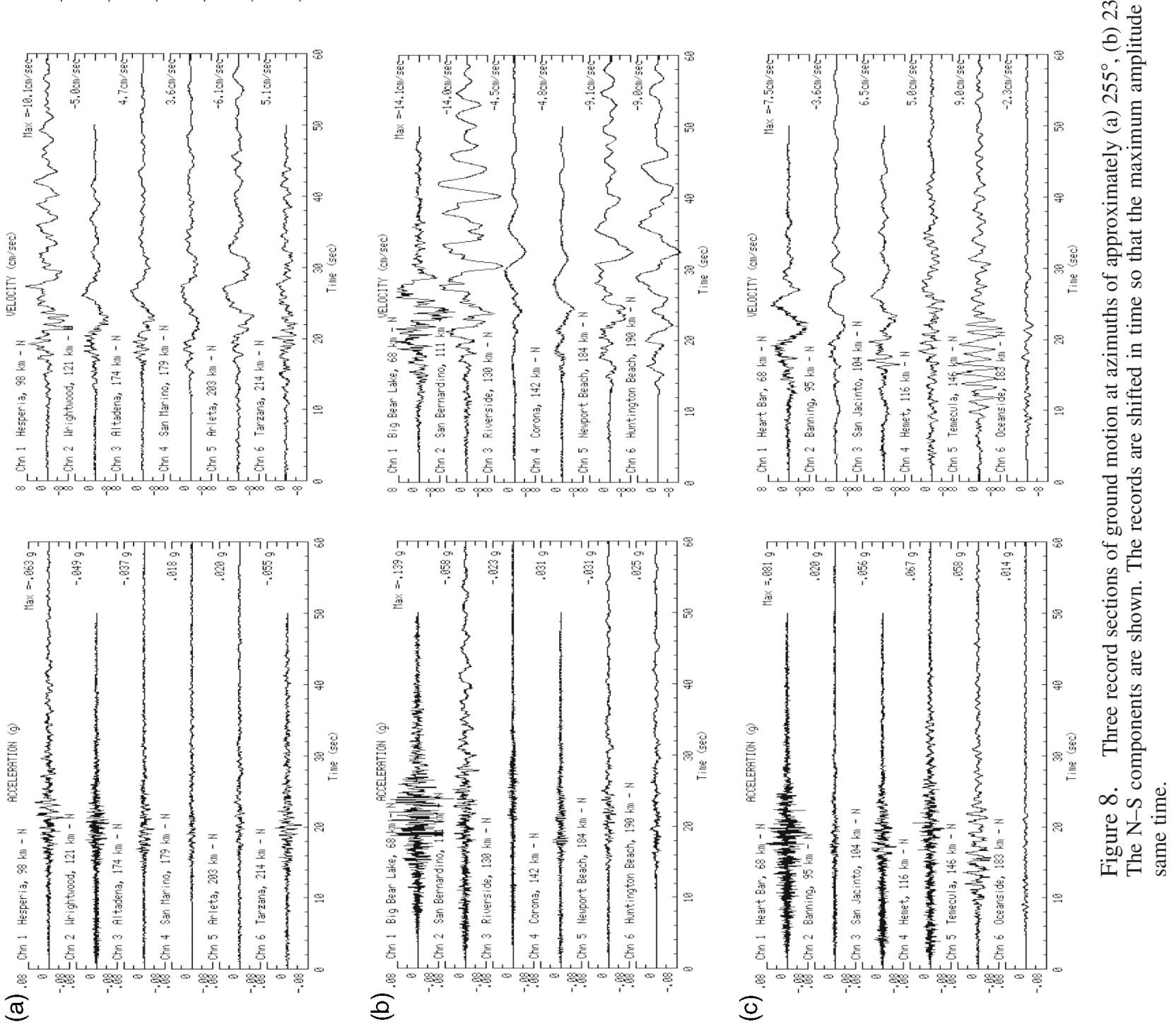
Table 3

Peak Horizontal Ground Motions in the Los Angeles Area during the Landers and Hector Mine Earthquakes

\begin{tabular}{|c|c|c|c|c|c|c|}
\hline \multirow[b]{2}{*}{$\begin{array}{l}\text { Station } \\
\text { ID }\end{array}$} & \multirow[b]{2}{*}{ Station Name } & \multicolumn{2}{|c|}{ Landers } & \multicolumn{2}{|c|}{ Hector Mine } & \multirow{2}{*}{$\begin{array}{c}\text { Landers/ } \\
\text { Hector } \\
\text { PGA } \\
\text { Ratio }\end{array}$} \\
\hline & & $\begin{array}{l}\text { Epi Dist } \\
(\mathrm{km})\end{array}$ & $\begin{array}{l}\text { PGA } \\
(g)\end{array}$ & $\begin{array}{l}\text { Epi Dist } \\
(\mathrm{km})\end{array}$ & $\begin{array}{l}\text { PGA } \\
(g)\end{array}$ & \\
\hline 24612 & Los Angeles-Pico \& Sentous & 169.4 & 0.034 & 193.9 & 0.019 & 1.79 \\
\hline 24611 & Los Angeles-Temple \& Hope & 167.6 & 0.031 & 191.2 & 0.027 & 1.15 \\
\hline 24605 & Los Angeles-7-story Univ. Hospital Grnds & 163.2 & 0.043 & 186.9 & 0.026 & 1.65 \\
\hline 24592 & Los Angeles-City Terrace & 160.8 & 0.058 & 184.8 & 0.038 & 1.53 \\
\hline 24400 & Los Angeles-Obregon Park & 161.6 & 0.065 & 186.0 & 0.033 & 1.97 \\
\hline \multirow{3}{*}{14403} & Los Angeles-116th St. School & 171.0 & 0.042 & 197.4 & 0.028 & 1.50 \\
\hline & Average & 165.6 & 0.0455 & 190.0 & 0.0285 & 1.60 \\
\hline & BJF97 & 165.6 & 0.0506 & 190.0 & 0.0409 & 1.24 \\
\hline
\end{tabular}

\section{Summary and Conclusions}

A large set of high-quality, mostly digital strong-motion records from more than 300 stations was collected from the Hector Mine earthquake, which occurred in the Mojave Desert. The closest station to the epicenter was approximately $25 \mathrm{~km}$ to the north and recorded a peak ground acceleration of $0.33 \mathrm{~g}$. The two other stations closest to the epicenter were located to the east and south at epicentral distances of about $50 \mathrm{~km}$; these recorded peak ground accelerations of about $0.2 g$.

Two main types of ground displacement waveforms were observed from the Hector Mine earthquake. The first type represents a relatively simple motion with a duration of 15 to $20 \mathrm{sec}$. This type of motion was observed everywhere, at epicentral distances from $50 \mathrm{~km}$ out to $240 \mathrm{~km}$, and may be associated with the source-generated $S$ wave. The second type of motion may have high amplitudes (more than $10 \mathrm{~cm}$ displacement) at epicentral distances of 160 to $220 \mathrm{~km} \mathrm{a}$ duration of about 40 seconds long; it has a sinusoidal type waveform with a predominant period of about 5 to $8 \mathrm{sec}$. The second type of motion appears to be typical for certain locations in the Los Angeles, Long Beach, San Bernardino, and EI Centro areas and most likely is created by Love waves (e.g., by multiple reflections of $S$ waves trapped in deep sedimentary basins).

In contrast to other data sets from large earthquakes in California, most of the Hector Mine strong-motion records were obtained at epicentral distances greater than $80 \mathrm{~km}$. In this specific set of data, any directivity effect is less significant than the effect of local and regional geology. This data set may be used to improve existing attenuation relationships, which usually have not had enough reliable strongmotion data for fault distances greater than $100 \mathrm{~km}$. Hector Mine peak ground accelerations (PGA) generally demonstrate reasonable agreement with the attenuation relationship developed by Boore, Joyner, and Fumal (Boore et al., 1997), which was developed for fault distances less than $100 \mathrm{~km}$ because adequate data were not available at greater distances.
Peak ground displacements (PGD), with relatively large amplitudes recorded at epicentral distances of 160 to 220 $\mathrm{km}$, do not demonstrate a normal attenuation pattern. Strongmotion data recorded in Los Angeles, Long Beach, and some other areas demonstrate significant, apparently basin-amplified wave amplitudes, especially at periods of 5 to $8 \mathrm{sec}$. This may reflect control by the sedimentary basin of the high-amplitude, long-period waves seen in the time domain.

Records from the Tarzana hill again demonstrated unusually high amplitudes of acceleration compared to the record at the foot of the hill. This site amplification effect has a clear directional character, with higher amplification along the component perpendicular to the strike of the hill. The source of the site amplification that produces large motions at Tarzana is still under investigation.

Comparisons of ground motions recorded from the $M_{\mathrm{w}}$ 7.1 Hector Mine and the $M_{\mathrm{w}}$ 7.3 Landers earthquakes indicate that the Landers records not only had higher amplitudes, but also had longer durations of ground motion. For the Los Angeles area, the Landers response spectra were on average two to three times higher than those of the Hector Mine event, except at periods of 6 to $7 \mathrm{sec}$, where they were about the same value.

Processed data from ground-response stations and a number of buildings and bridges were made available quickly after the earthquake at ftp://ftp.consrv.ca.gov/pub/ dmg/csmip/Hector_Mine/(now HectorMine 99). Additional records of the USGS, CDMG, and other networks continue to be collected and verified and will be put on the TriNet/ CISN Web site (http://www.cisn.org).

\section{Acknowledgments}

The TriNet networks extend their appreciation to the individuals and organizations that have permitted and cooperated in the installation of seismic strong-motion equipment on their property. The records presented in this paper were made possible through the efforts of technicians who installed and maintained these stations. The authors thank Bill Bryant for providing a digital file of the surface rupture associated with the Hector Mine earthquake. The authors also thank Tianquing Cao for assistance in digitizing and data processing, and Jim Agnew and Hamid Haddadi for 
(a)

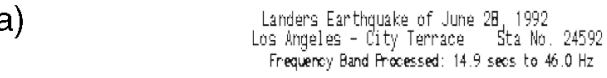

MCCELERRT ION
.05 - thin 1: in on

0 -

5 - Wha : : U

Elimi t: tip

05 - 05 tha 3: 180 dag

10 - ihin 1: ind of

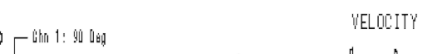

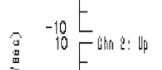

3.

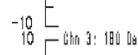

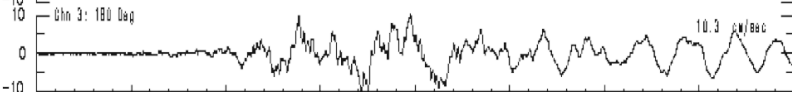

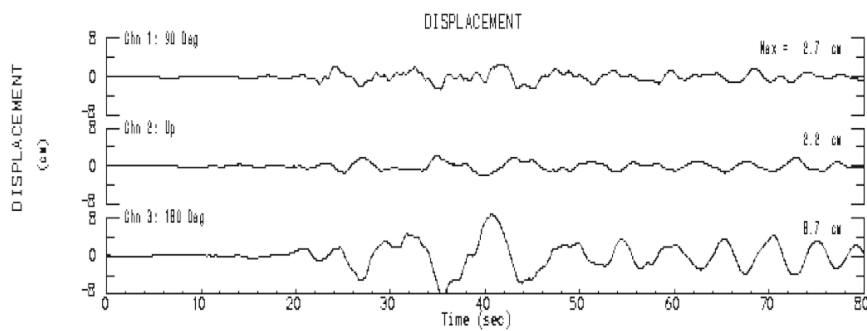

(b)

$$
\begin{aligned}
& \text { Landers Ear thquake of Jume 28, } 1992
\end{aligned}
$$

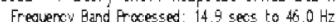
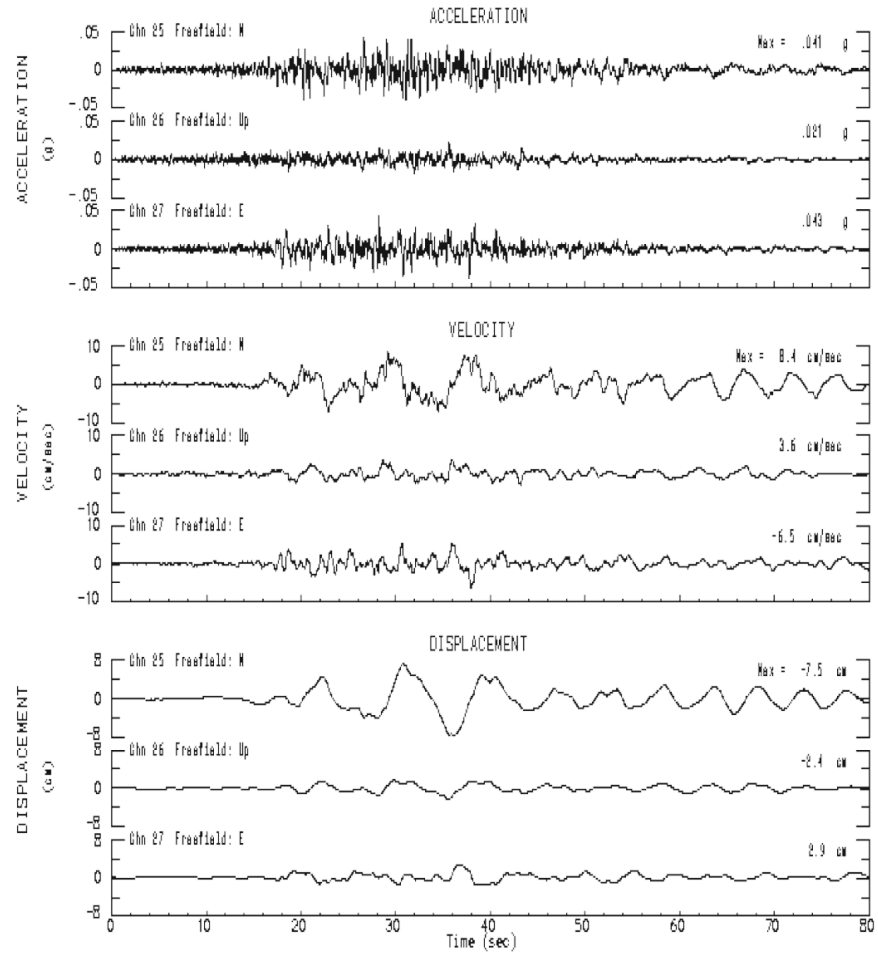

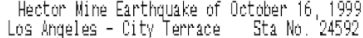

Frequency Hand Frocessed: 14.9 secs to $46.0 \mathrm{~Hz}$

MCCELERLTION
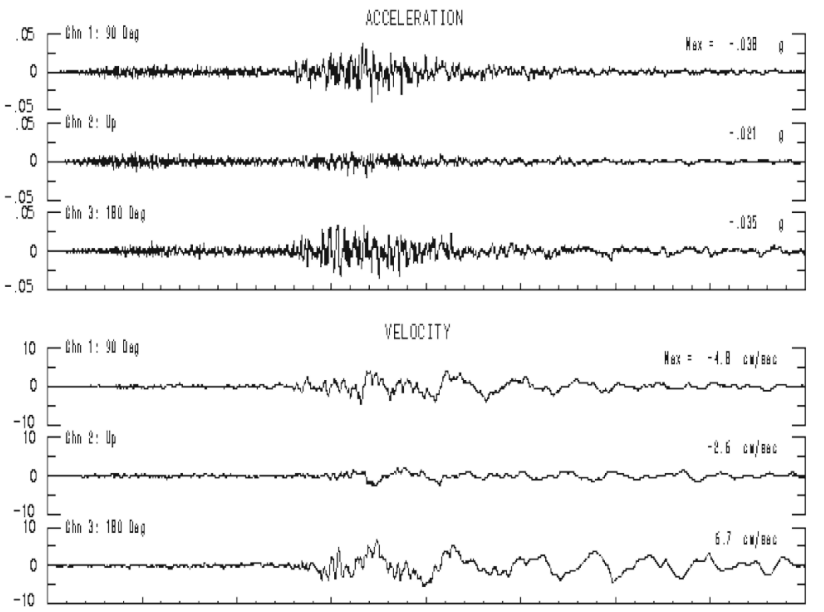

DIIFLLCIENENT

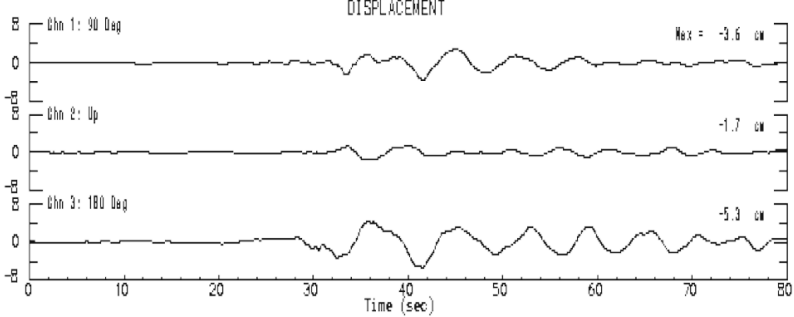

Hector Hine Eanthquake of Dotoberer 16,1990 Los drigeles - 7-story uniw. Hospital Gernds 5ta No. 24605 Frequency Gand Froxessed: 14.9 secs to $45.0 \mathrm{~Hz}$

MCCELER MT ION

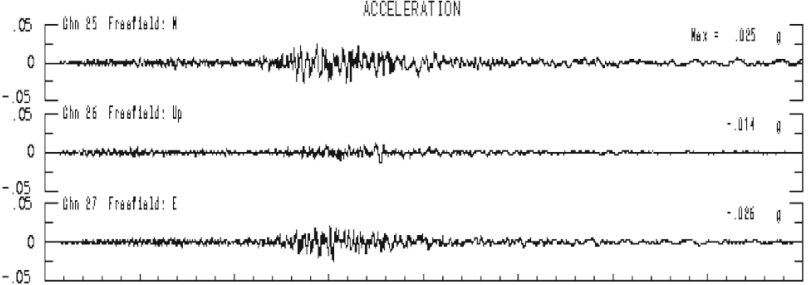

VELOCITY
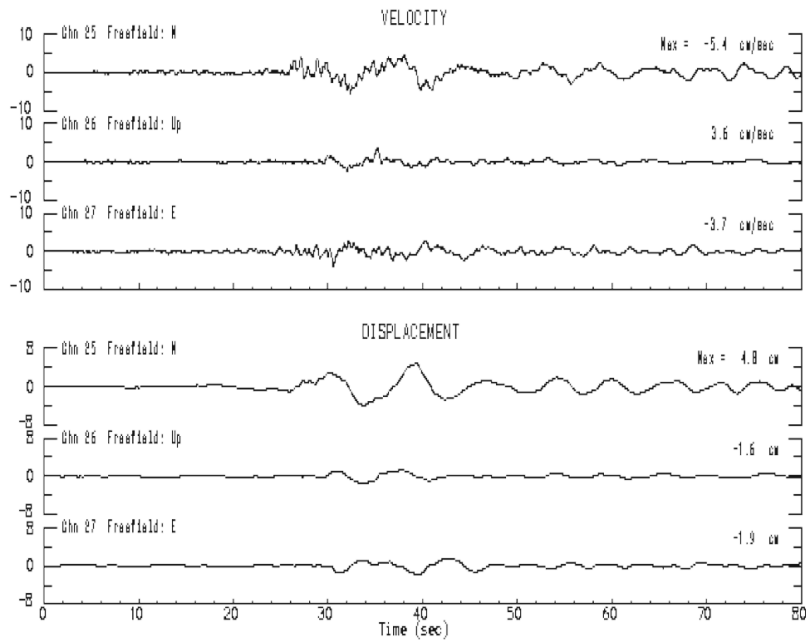

Figure 9. Strong ground motions recorded at two stations in the Los Angeles area during the Hector Mine earthquake and the Landers earthquake of 1992: (a) Los Angeles-City Terrace; (b) Los Angeles-University Hospital Grounds. 
Ratio of Hector to Landers Earthquake Response Spectra

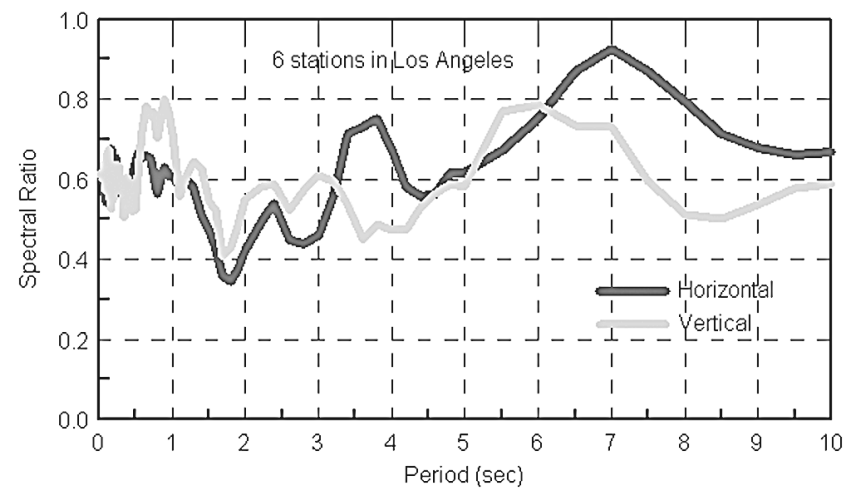

Figure 10. Ratios of the average response spectra (5\% damped) for the six stations in the Los Angeles area comparing the Hector Mine earthquake to the Landers earthquake of 1992.

reviewing the manuscript. The manuscript benefited from comments of Michael Rymer and two anonymous reviewers.

\section{References}

Boore, D. M., W. B. Joyner, and T. E. Fumal (1993). Estimation of response spectra and peak accelerations from western North American earthquakes: an interim report, U.S. Geol. Surv. Open-File Rept. 93-509.

Boore, D. M., W. B. Joyner, and T. E. Fumal (1997). Equations for estimating horizontal response spectra and peak acceleration from western North American earthquakes: a summary of recent work, Seism. Res. Lett. 68, no. 1, 128-153.

Bouchon, M., and J. S. Barker (1996). Seismic response of a hill: the example of Tarzana, California, Bull. Seism. Soc. Am. 86, no. 1A, 6672.

Catchings, R. D., and W. H. K. Lee (1996). Shallow velocity structure and Poisson's ratio at Tarzana, California, strong-motion accelerometer site, Bull. Seism. Soc. Am. 86, 1704-1713.

Cramer, C. H., and R. B. Darragh (1994). Peak accelerations from the 1992 Landers and Big Bear, California, earthquakes, Bull. Seism. Soc. Am. 84, no. 3, 589-595.

Darragh, R., V. Graizer, and A. Shakal (1997). Site characterization and site response effects at CSMIP stations: Tarzana and La Cienega near the Santa Monica freeway (1-10). California Strong Motion Instrumentation Program, OSMS 96-07, Sacramento, California, 15 January 1997, 262 pp.

Darragh, R., V. Graizer, and A. Shakal (1998). Tarzana, California: site response and characterization, in Proceedings of the NEHRP Conference and Workshop on Research on the Northridge, California Earthquake of January 17, 1994, Vol. II, 323-330.

Dreger, D., and A. Kaverina (2000a). Seismic remote sensing for the earthquake source process and near-source strong shaking: a case study of the October 16, 1999 Hector Mine earthquake, Geophys. Res. Lett. 27, no. 13, 1941-1944.

Dreger, D., and A. Kaverina (2000b). Source process of the October 16, 1999 Hector Mine earthquake, in Berkeley Seismological Laboratory Annual Report, July 1999-June 2000, 100-101.

Graizer V. M., T. Cao, A. F. Shakal, and P. Hipley (2000a). Data from downhole arrays instrumented by the California Strong Motion Instrumentation Program in studies of site amplification effects, in Proceedings [CD-ROM] of the Sixth International Conference on Seismic Zonation (61CSZ), Earthquake Engineering Research Institute, Palm Springs, California, 12-15 November 2000.
Graizer V. M., A. F. Shakai, and P. Hipley (2000b). Recent data recorded from downhole geotechnical arrays, in Proceedings of SMIP2000 Seminar on Utilization of Strong-Motion Data, Sacramento, California, 14 September 2000, 23-38.

Hartzell, S., A. Leeds, A. Frankel, and J. Michael (1996). Site response for urban Los Angeles using aftershocks of the Northridge earthquake, Bull. Seism. Soc. Am. 86, no. 1B, S168-S192.

Hauksson, E., L. M. Jones, and K. Hutton (2002). The $1999 M_{\mathrm{w}} 7.1$ Hector Mine, California, earthquake sequence: complex conjugate strike-slip faulting, Bull. Seism. Soc. Am. 92, 1154-1170 (this issue).

Jennings, C. W. (1977). Geologic map of California. Scale 1:750,000. California Division of Mines and Geology, Sacramento, California 95814.

Jennings, C. W. (Compiler) (1994). Fault activity map of California and adjacent areas, California Department of Conservation, Division of Mines and Geology, Geologic Data Series No. 6, scale 1:750,000.

Mori, J., H. Kanamori, J. Davis, E. Hauksson, R. Clayton, T. Heaton, L. Jones, and A. Shakal (1999). Major improvements in progress for southern California earthquake monitoring, EOS 79, 217-221.

Park, S., and S. Elrick (1998) Prediction of shear-wave velocities in southern California using surface geology, Bull. Seism. Soc. Am. 88, no. 3, $677-685$

Scientists of the U.S. Geological Survey, Southern California Earthquake Center, and California Division of Mines and Geology (2000). Preliminary report on the 16 October 1999 M 7.1 Hector Mine, California, earthquake, Seism. Res. Lett. 71, no. 1, 11-23.

Shakal, A., M. Huang, and T. Cao (1988). The Whittier Narrows, California earthquake of October 1, 1987: CSMIP strong motion data, Earthquake Spectra 4, 75-100.

Shakal, A., M. Huang, T. Cao, R. Sherburne, R. Sydnor, P. Fung, P. Malhotra, C. Cramer, F. Su, R. Darragh, and J. Warnpole (1992). CSMIP strong-motion records from the Landers, California earthquake of 28 June 1992. California Strong Motion Instrumentation Program, OSMS 92-09, Sacramento, California, August 5, 1992, 330 pp.

Shakal, A. F., M. J. Huang and R. B. Darragh (1996). Interpretation of significant ground-response and structure strong motions recorded during the 1994 Northridge earthquake, Bull. Seism. Soc. Am. 86, no. 1B, S231-S246.

Shakal, A., M. Huang, R. Darragh, T. Cao, R. Sherburne, P. Malhotra, C. Cramer, R. Sydnor, V. Graizer, G. Maldonado, C. Petersen and J. Wampole (1994). CSMIP strong-motion records from the Northridge, California earthquake of 17 January 1994, California Strong Motion Instrumentation Program, OSMS 94-07, Sacramento, California, 18 February 1994, 308 pp.

Spudich, P., M. Hellweg, and W. H. K. Lee (1996). Directional topographic site response at Tarzana observed in aftershocks of the 1994 Northridge, California, earthquake: implications for mainshock motions, Bull. Seism. Soc. Am. 86, no. 1B, S193-S208.

Treiman, J. A., K. J. Kendrick, W. A. Bryant, T. K. Rockwell, and S. F. McGill (2002). Primary surface rupture associated with the $M_{\mathrm{w}} 7.1$ 16 October 1999 Hector Mine earthquake, San Bernardino County, California, Bull. Seism. Soc. Am. 92, 1171-1191 (this issue).

Vidale, J. E., O. Bonamassa, and H. Houston (1991). Directional site resonances observed from the 1 October 1987 Whittier Narrows, California, earthquake and the 4 October aftershock, Earthquake Spectra 7, 107-125.

Wald, D. J., and T. H. Heaton (1994). Spatial and temporal distribution of slip for the 1992 Landers, California, earthquake, Bull. Seism. Soc. Am. 84, no. 3, 668-691.

California Division of Mines and Geology

Strong Motion Instrumentation Program

801 K Street, MS 13-35

Sacramento, California 95814

vgraizer@consrv.ca.gov

(V.G., A.S.) 
California Division of Mines and Geology and

California Institute of Technology

Pasadena, California 91125

(C.S.)

California Institute of Technology

Pasadena, California 91125

(E.H., J.P.)
U.S. Geological Survey

Pasadena, California 91106

(L.J.)

Manuscript received 23 January 2001. 